\title{
Upwind 2MW Horizontal Axis Wind Turbine Tower Design and Analysis
}

\author{
Gizachew Dereje Tsega ${ }^{1,}$, , Belete Sirahbizu Yigezu² \\ ${ }^{1}$ Mechanical Engineering, University of Gondar Institute of Technology School of Mechanical Engineering, Gondar, Ethiopia \\ ${ }^{2}$ Mechanical Engineering, College of Electrical and Mechanical Engineering University-Industry Linkage Directorate Director, Addis Ababa \\ Science and Technology University, Addis Ababa, Ethiopia
}

\section{Email address:}

anasamu120@gmail.com (G. D. Tsega),belete.sirhabizu@aastu.edu.et(B. S. Yigezu)

*Corresponding author

\section{To cite this article:}

G Gizachew Dereje Tsega, Belete Sirahbizu Yigezu. Upwind 2MW Horizontal Axis Wind Turbine Tower Design and Analysis. Automation, Control and Intelligent Systems. Vol. 7, No. 5, 2019, pp. 111-131. doi: 10.11648/j.acis.20190705.11

Received: June 6, 2019; Accepted: August 12, 2019; Published: January 30, 2020

\begin{abstract}
Wind energy is one of the quickest growing renewable energies in the world due to era of wind energy is smooth and non-polluting; it does now not produce any byproducts dangerous to the environment. Large scale machines are in particular nicely appropriate for wind energy. The fee of foundations doesn't upward push in share to the dimensions of the device, and protection costs are largely impartial of the size of the system. In areas where it is difficult to find sites for more than a single turbine, a large turbine with a tall tower uses the existing wind resource more efficiently. Different subcomponents are designed depend on the purpose of the turbines among these the tower of a wind turbine helps the nacelle and the rotor and affords the necessary elevation of the rotor to hold it clear off the floor and produce it as much as the level where the wind sources are. The towers for large wind turbines are typically made from steel; however concrete towers are every so often used. The tower is normally connected to its helping basis by using a bolted flange connection or a weld. The tower constitutes a low-generation aspect whose layout is easy to optimize, and which therefore for the duration of the layout manner lends itself easily as an item for possible fee discount. This may additionally are available in useful because the fee of a tower typically establishment a sizeable a part of the entire fee of a wind turbine. The design and analysis of the tower focused on large wind turbines. It examines the result of loading on the tower, the optimum tower height and the verification of safety against bending and buckling. The buckling of $2 \mathrm{MW}$ horizontal axis wind turbine tower tube with tower base diameter of $3.9 \mathrm{~m}$, top tower diameter of $2 \mathrm{~m}$ and length of $80 \mathrm{~m}$ is studied by theoretical analysis and numerical simulation by using ANSYS and MATLAB software. Based on this study the results are calculated based on theoretical and FEM method and their error is shown, buckling modes and vibrational analysis are done, shear and bending diagrams are shown, extreme loading conditions are also shown.
\end{abstract}

Keywords: Renewable Energy, Wind Energy, Horizontal-Axis Wind Turbine, Aerodynamics, Tubular Tower

\section{Introduction}

Renewable energy assets are the energy sources, which are not spoiled when their energy is harnessed. Human use of renewable strength calls for technologies that harness herbal phenomena which includes daylight, wind, waves, water glide, and natural processes including natural hydrogen manufacturing and geothermal warmth. Amongst the above stated assets of electricity there was quite a few development within the generation for harnessing electricity from the wind
$[1]$.

The energy transferred to the rotor by using the wind relies upon on the air density, the swept region of the rotor and wind speed. Blade is the important thing element to capture wind power. It performs a vital position inside the complete wind turbine. Turbine energy production depends at the interplay among the rotor and the wind [2].

A system which converts the power within the wind into energy is wind turbine; which contrasts a windmill that is a device that converts the wind's power into mechanical power. Wind power, as an opportunity to fossil fuels, is ample, 
renewable, and significantly circulated, smooth, produces no greenhouse gas emissions throughout operation and makes use of little land [16]. If the efficiency of a wind turbine is expanded, then more electricity may be generated as a consequence reducing the need for pricey energy era that causes pollution. Ever because the seventh century, people had been making use of wind to make their lives less complicated [3].

Wind turbines are very complicated systems that strongly couples mechanical operation, digital controls, structural and geotechnical support structures all even as being subjected to uncertain forces from nature.

\section{Problem Definition}

Since the tower of a wind turbine helps the nacelle and the rotor and provides the necessary elevation of the rotor to preserve it clear off the ground and convey it as much as the level in which the wind assets are perfect to generate the desired electric energy. This function of a tower leads failures due to forces evolved through the blade as thrust pressure, the load of the nacelle, weight of rotor and other structures. In order to prevent this failures a proper design and evaluation of a tower is substantial and a choice-much less approach.

\section{Background}

The tower is crucial component of the horizontal-axis turbine, a fact which can be both an advantage and a disadvantage. As the peak of the tower increases, transportation, assembly and erection of the tower and servicing of the additives additionally come to be an increasing number of difficult and high-priced. On the alternative hand, the specific energy yield of the rotor additionally increases with tower peak. Theoretically, the most beneficial tower height lies on the point wherein the two growth functions of production value and energy yield intersect. Unfortunately, this point of intersection can't be laid out in any commonly applicable shape.

In larger turbines, construction prices rise greater rapidly with growing tower peak than in small turbines. An even extra role is performed through the selection of site. At inland sites, i.e. in regions with excessive degree of surface roughness, the wind speed increases greater slowly with height than at shore based totally sites. Higher towers will, consequently, show higher returns here than, as an instance, in offshore applications wherein the opposite effect is located. In inland regions, large wind turbines with tower heights of $80 \mathrm{~m}$ and extra are a decisive thing for the monetary use of the wind potential.

Next to its peak, the second one maximum important layout parameter of a tower is its stiffness. Establishing the primary natural bending frequency in the right way is a crucial mission in the design. This determines the material required and, in the end, the construction prices. The intention of the tower design is to reap the favored tower height with the required stiffness at the bottom possible production cost.

The transportation and the erection technique are developing into an increasing problem for the contemporary era of multi-megawatt wind turbines. Tower heights of extra than $100 \mathrm{~m}$ and tower head weights of several hundred tons require a diameter at the tower base of greater than five meters, with the result that avenue transportation will now not be viable. This turns into a strong incentive to locate modern solutions within the tower design.

The materials available for the construction are steel or concrete. Design variety from lattice constructions to free standing steel tubular towers up to big concrete systems. The technical necessities posed by means of the overall system may be met by almost any version however the financial optimal is only done with the aid of correctly matching the chosen tower design to the requirements set. This shows virtually that, although the tower of a wind turbine can be visible as a conventional structure when taken into consideration with the aid of itself, its layout also calls for a considerable amount of understanding of the general machine and its application. Apart from those purposeful elements, it have to now not be disregarded that the tower, even greater so than the nacelle, determines the outward appearance of a wind turbine. Due attention have to, therefore, be accorded aesthetics, despite the fact that this means some extra charges.

\section{Methodology and Design Procedure}

The principal motive of this paper is to estimate the effect of thrust loads on the tower. The consequences of loading to be taken into consideration are bending and buckling from which the layout needs to be secure. This work also offers a layout that optimizes the height and the mass of the tower. The technique of this paper is specifically based on literature overview of published studies papers and books regarding the loadings carried out, and their different outcomes. The purpose of this part is to acquire the vital formulas to determine the allowable stress thru calculating the bending strain and the neighborhood buckling. This additionally consists of the height specifications of the tower at which it have to optimally perform.

Based at the given certain design parameters, the evaluation and implementations are carried out especially via by using the usage of MATLAB ${ }^{\circledR} 2018$ b, ANSYS ${ }^{\circledR} 19.2$ and SOLIDWORKS® 2019.

Academic sources are reviewed for in addition optimization strategies and advanced calculations. To achieve this examine design strategies consciousness on:

1. Defining loads.

2. Perform analysis.

3. Derive dimensions and details.

Considering design loads such as:

1. Dead load or self-weight.

2. Direct wind pressure (applied as static).

3. Turbine load (applied as dynamic or amplified static).

4. External loads (natural disaster). 


\section{Wind Turbine}

A wind turbine uses the aerodynamic force of the lift to rotate a shaft which in turn enables within the conversion of mechanical energy to electricity via a generator.

Wind turbine generator which converts the kinetic energy of the wind into mechanical shaft energy to drive a generator that in turn produces electrical energy. A wind turbine (WT) consists of five foremost factors:

1. Rotor made up of rotor blades that use aerodynamic lift to convert wind energy into mechanical energy.

2. A rotor bearing fixed on a structure that causes a defined rotation of the rotor and leads to conversion of the aerodynamic wind energy into a rotational shaft torque. A yaw system maintains the horizontal rotor axis pointing upstream into the wind.

3. A power conversion system that converts the low-speed rotational energy into suitable shaft power to drive an electrical generator.

4. A tower and foundation structure to support the rotor and generator system at a height that harvests the most amount of energy for an acceptable capital cost.

5. An electrical power distribution system that supplies the energy to the consumer in compliance with local grid code and system requirements.

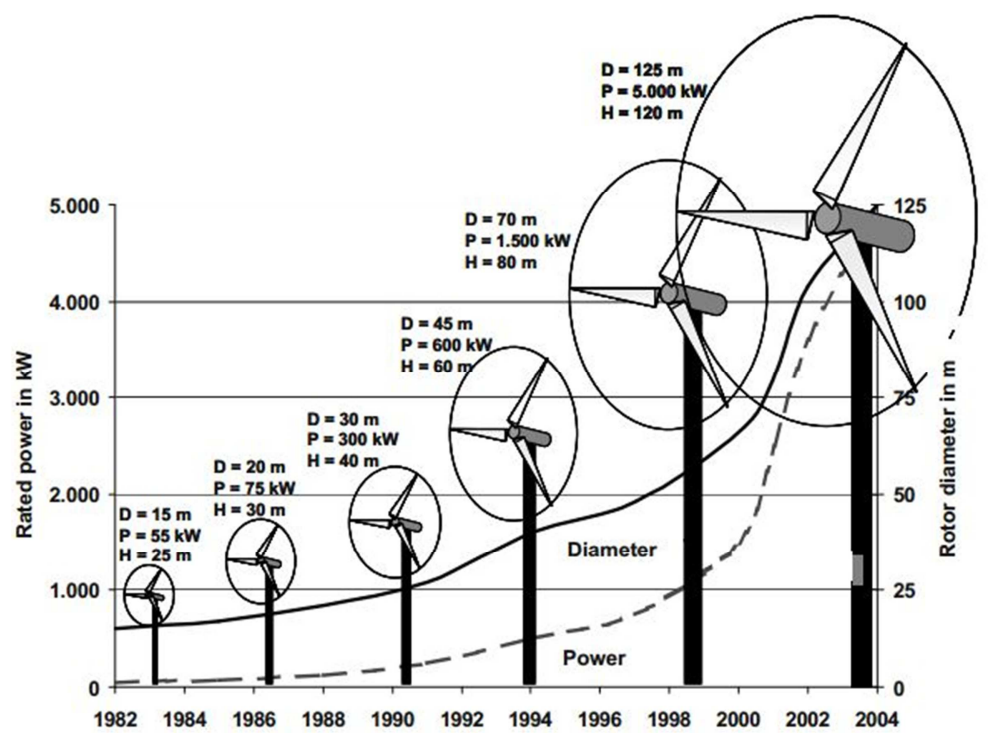

Figure 1. Representative size, height, and diameter of wind turbines.

The principal subsystems of a typical horizontal-axis wind turbine as shown in Figure include [2]:

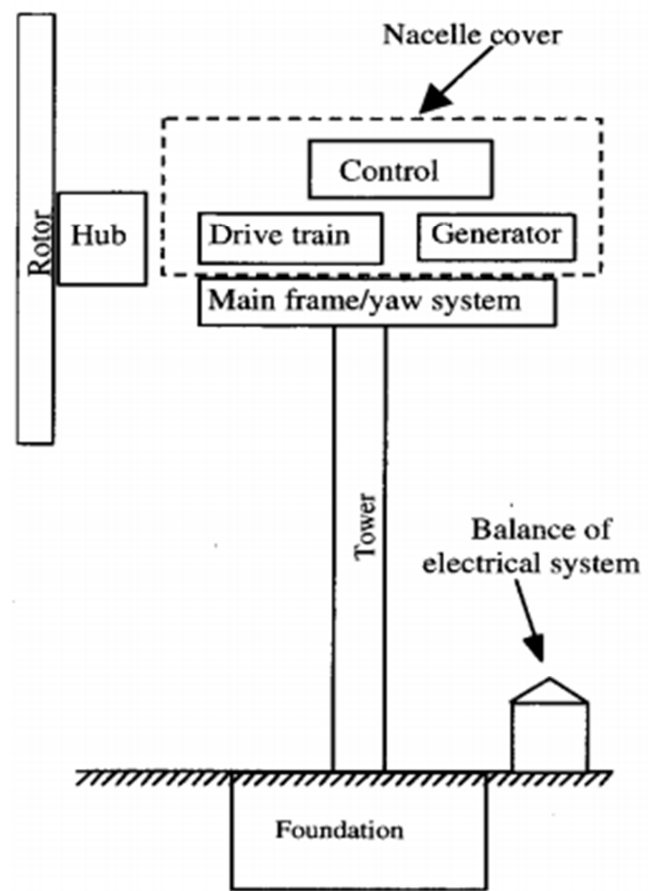

Figure 2. Major components of a horizontal axis wind turbine. 


\section{The Physics of Wind Energy}

\subsection{Energy in the Wind}

The power of the wind that flows at a velocity $v$ through an area $A$ is

$$
P_{\text {wind }}=\frac{1}{2} \rho A v^{3}
$$

It is proportional to the air density $\rho$, the cross sectional area $A$ (perpendicular to $v$ ) and the power of the wind velocity $v$. or the power of the wind velocity is explained the power in the wind $P$ wind is the kinetic energy of the air mass $m$, passing through the area $A$ in a given time.

$$
E=\frac{1}{2} m V^{2}
$$

Since the resulting mass flow

$$
=A \rho \frac{d x}{d t}=\rho A v
$$

it is proportional to the wind velocity, the power (energy per unit of time) is expressed by

$$
P_{\text {wind }}=\dot{\mathrm{E}}=\frac{1}{2} \quad v^{2}=\frac{1}{2} \rho A v^{3}
$$

Table 1. Typical wind turbine sizes as design data.

\begin{tabular}{lll}
\hline Tower height $(\mathbf{m})$ & Rotor diameter $(\mathbf{m})$ & Rated power $(\mathbf{k w})$ \\
\hline 22 & 21 & 55 \\
31 & 30 & 225 \\
35 & 35 & 450 \\
$35-40$ & $41-44$ & 500 \\
44 & 43 & 600 \\
50 & 48 & 750 \\
50 & 54 & 1000 \\
60 & 58 & 1500 \\
$64-80$ & $72-76$ & 2000 \\
85 & 115 & 5000 \\
\hline
\end{tabular}

The swept area

$$
A=\pi \frac{D^{2}}{4}=\pi R^{2}
$$

Take swept radius as 36 from table 1 .

$$
A=4071.5 \mathrm{~m}^{2}
$$

Power in the air

$$
\begin{gathered}
p_{w}=0.5 \rho A v^{3} \\
p_{w}=430275.6 \text { watts } \\
=4.3 \mathrm{MW}
\end{gathered}
$$

but at different atitude $H$

$$
\begin{gathered}
\rho=\rho_{0}-1.994 * 10^{-4} * H \\
1.225-1.994 * 10^{-4} * 80 \\
=1.210672 \mathrm{~kg} / \mathrm{m}^{3}
\end{gathered}
$$

$$
p_{w}=4258872.905 \text { watts }
$$

$$
=4.25 \mathrm{MW}
$$

\subsection{Power of Blade}

By taking the theoretical efficiency range of wind power from range and taking 0.47

$$
\begin{gathered}
p_{\text {blade }}=c_{p} * p_{w} \eta \\
p_{\text {blade }}=2.0825 \mathrm{MW}
\end{gathered}
$$

The efficiency of energy transformation in turbine

$$
\begin{aligned}
& \eta=\frac{p_{\text {blade }}}{p_{w}} \\
& \eta=0.4755 \approx 0.48 \\
& \omega=\frac{\lambda v}{R}=7 * \frac{12}{36} \\
& =2.33 \frac{\mathrm{rad}}{\mathrm{s}} \\
& \omega=\frac{2 \pi N}{60} \\
& N=\omega * \frac{60}{2 \pi}=22.28 \approx 22 \mathrm{rpm} \\
& \text { Rotor torque }=\frac{p_{\text {blade }}}{\omega} \\
& \text { Rotor torque }=\frac{2.0825 \mathrm{MW}}{2.33} \\
& =894\left(\frac{K N}{m}\right) \\
& \text { total thrust }=\frac{1}{2} \rho A v^{2} \\
& =0.5 * 1.210672 * 4071.5 * 12^{2} \\
& =354.9 \mathrm{KN} \\
& \text { thurst }=C_{T} * \text { total thrust } \\
& =\frac{C_{p}}{\lambda} * \text { total thrust } \\
& =0.063 * 354.9 K N \\
& =22359.08 \mathrm{~N}
\end{aligned}
$$

\section{Tower}

\subsection{Tower Configurations}

The oldest types of "wind turbines", the windmills, had no towers but millhouses. These were low in height in relation to the rotor diameter and of voluminous construction in accordance with their function as a work space, thus also providing for the necessary stiffness. Soon, however, the advantage of increased height was recognized and the 
millhouses became more slender and more tower-like. But it is only in modern-day constructions, first in the small American wind turbines and then later in the first powergenerating wind power stations, that "masts" or "towers" were used, the sole function of which lay in supporting the rotor and the mechanical components of the tower head. As a consequence of this development, designs and materials for towers increased in variety. Steel and concrete took the place of the wood construction of the millhouses. In the early years of the development of modern wind energy technology, the most varied tower designs were tried out and tested but in the course of time, the range has been narrowed down to freestanding designs, mainly of steel and more rarely of concrete.

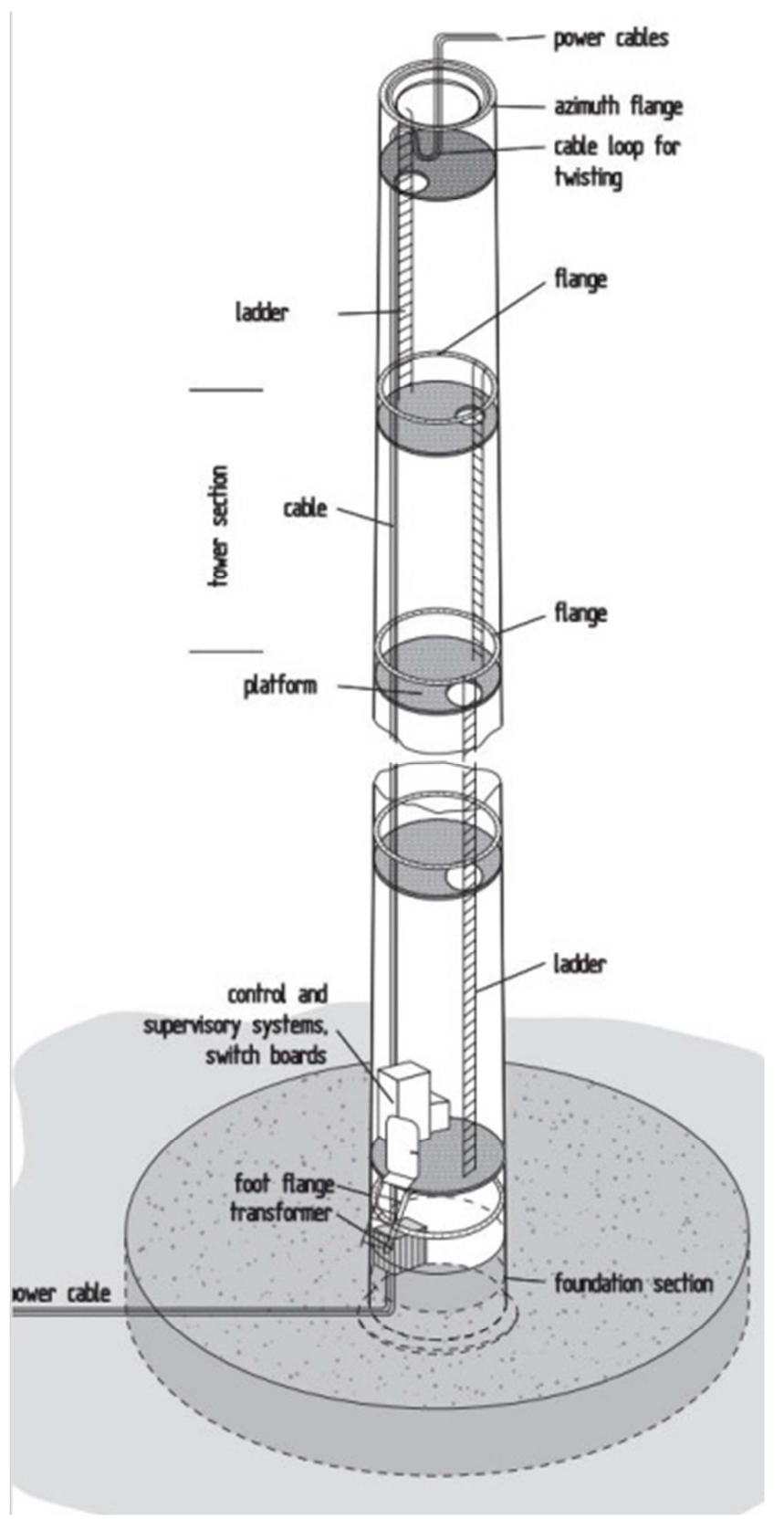

Figure 3. Tabular steel tower with installation of large wind turbine.

\subsection{Strength and Stiffness Design}

The dimensioning of a tower is decided via some strength and stiffness necessities. Factors to be taken into consideration are the breaking power required for surviving intense wind speeds, the fatigue strength required for 20 or 30 years of operation and the stiffness with respect to the vibrational behavior.

The stiffness requirement is derived from the chosen vibrational concept of the turbine as a whole. It is generally focused on the requirement for a particular first natural bending frequency, even though other natural frequencies, and particularly the natural torsion frequency, must be checked with regard to the dynamics of the yaw system of the turbine.

\subsection{Breaking Strength}

The static load is determined by the tower-head weight, the tower's own weight, and the aerodynamic rotor thrust. In turbines with blade pitch control, rotor thrust is generally at its highest level when the rotor is running at its rated speed. It can, however, be surpassed by the wind load during rotor standstill at extreme wind speeds. The maximum bending moment distribution at the tower is obtained with rotors without blade pitch control (stall-controlled turbines) or when the worst rotor blade position is demanded for a particular load case. In the standard case, the question of breaking load will be reduced to that of the bending moment acting on the tower base.

\subsection{Fatigue Loading}

The dynamic loading caused by the rotor thrust during operation has a definite impact on the fatigue life of slender towers. Additional loads caused by the vibrational behavior in cases of resonance must also be taken into consideration. Hence a purely static stress analysis, commonly required by the building authorities for conventional buildings, is not appropriate for all tower designs of a wind turbine.

\subsection{Buckling Strength}

One important criterion which plays a role at least for thinwalled steel tubular towers with a low natural bending frequency below the $1 P$ excitations is the resistance to local buckling of the tube wall. As a result of the increasing weight optimization in modern steel tubular towers, the buckling strength frequently becomes the determining dimensioning factor for the required wall thicknesses.

Buckling is the most common mode of failure of turbine towers it is caused due to extreme loading or fatigue loads. The trend in tower design is to reduce the thickness of tabular steel towers to make economical also designing them susceptible to buckling failure is the key point. 


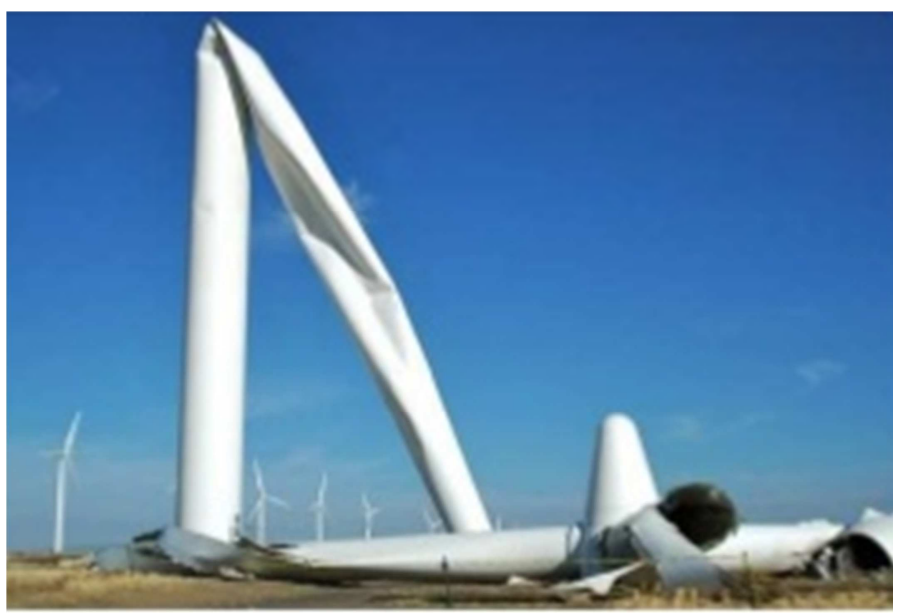

Figure 4. Large scale wind turbine failure due to buckling.

Tower stiffness is characterized by several natural frequencies, but only the first and the second natural bending frequency and the first natural torsion frequency are of any practical significance. In most towers, the first natural torsion frequency is much higher than the first natural bending frequency. The torsion frequency of free-standing steel tubular towers is approximately three times higher if their diameter/wall thickness ratio lies within normal limits. It is, therefore, sufficient to use the first natural bending frequency for obtaining a rough overview. With a given tower height and head weight, the tower must be designed in such a way that the required first natural bending frequency is reached. A stiff tower design is always a simpler and safer solution with regard to vibrational behavior, but the mass of the tower required to achieve this becomes very high. In wind turbines with tower heights of more than $80 \mathrm{~m}$, a stiff tower design can, therefore, no longer be realized in practice. For economic reasons, the stiffness should be kept as low as technically feasible.

For simple tower geometries, for example a cylindrical steel tube, dimensioning models were developed which permit the required wall thickness to be calculated by using relatively simple formulae, on the basis of the said load cases with a given height, tower head mass and the chosen stiffness concept of the wind turbine [2]. These models are mainly suited to demonstrating the influence of the dimensioning parameters, thus helping to understand their significance with regard to tower optimization. In reality, the calculated masses are often lower. Manufacturers increasingly tend to favor more complicated designs such as wall thickness varying in stages with diameter, or weight-optimized tapered shapes to minimize the tower mass and thus the costs.

\subsection{Tower Types}
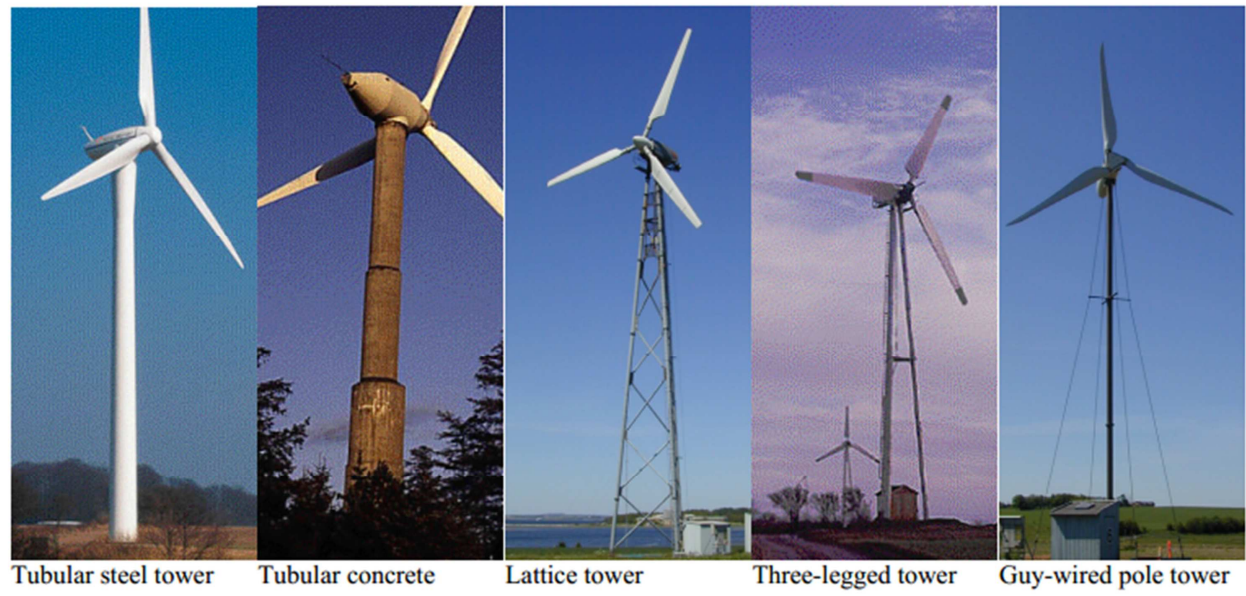

Figure 5. Tower structures.

Table 2. Main types of towers.

\begin{tabular}{lll}
\hline Type & Main advantages & Main disadvantages \\
\hline Monopole & Looks aesthetically nice & Uses more steel. More costly to produce and transport. \\
Guyed & Reduced cost. Minimum material & Not suitable for urban installation \\
Sectional lattice & Reduced transportation cost & Short lifetime. Joints corrodes \\
Tabular lattice & Simple to produce. Light weight & Electrical cables are less protected \\
\hline
\end{tabular}




\section{Optimum Tower Height}

The power output varies depending on height of the wind turbine. Typically, tower height $h$, is in a range between 10 and 50 meters. The values for $h$ are directly associated with the change of wind speed depending on altitude. But before expressing the dependency of height on the mean wind speed, it is important to follow some rules of thumb provided by Mick Sagrillo for siting wind turbines.

Due to reduced ground drag, wind speed increases proportionally with height. Minimum tower height should be at least higher by 10 meters than anything in the surrounding 150 meters. - Rougher terrain produces more disruption of the wind. In the lower layers of the atmosphere, the wind speed is more affected due to friction with the surface of the Earth. During siting, the roughness of the terrain, which represents the impact of obstacles and surrounding contours, must be taken into account. The rougher the ground surface is, the more disrupted and slowed down is the wind. Another rule of thumb that has to be respected is that the tip of the blade should be at least $15 \mathrm{~m}$ above the ground

$$
\frac{D}{2}+15 \leq H_{h u b}
$$

Where $D$ is the rotor diameter and $H h u b$ is the height of the hub.

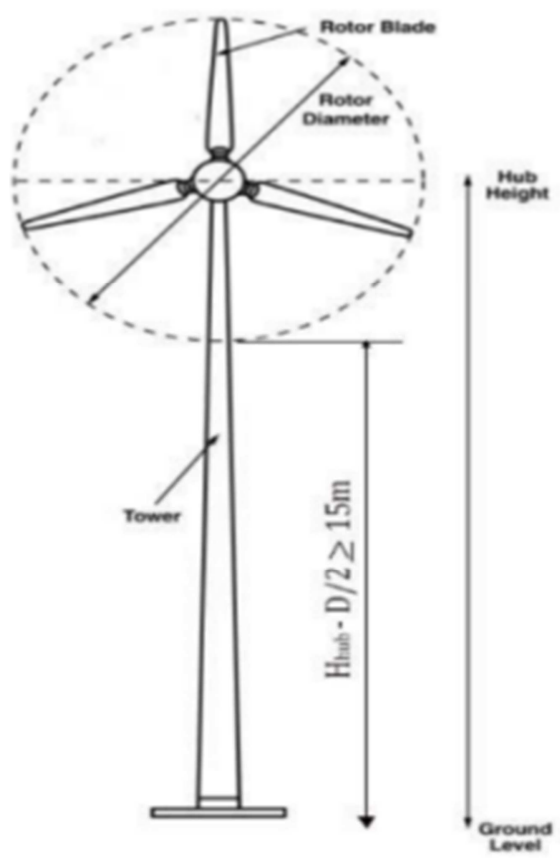

Figure 6. Minimum distance between tip blade and the ground.

\section{Design Loads}

Regardless of which analysis approach is used for these calculations, the loads should be calculated from a model, in which the tower properties (geometry, materials, and stiffness) are in agreement with the ones used in the final design. Thus, the design of the tower may demand an iterative procedure to get from an initial design to the final design with the correct stiffness. The design loads for fatigue are to be determined by calculations, which are to be supplemented and verified by actual measurements from a prototype turbine. Since load measurements cannot be made until the turbine has been designed and constructed, it is recommended to apply an additional partial safety factor of 4.62 on fatigue loads until measurements are carried out and become available. The use of an additional safety factor as an extra precaution is meant to avoid a major redesign in the event of increased design loads.

The extreme design loads can only be determined by calculations, because these loads cannot be measured due to the long recurrence period between events. When designing a turbine with fixed speed, the frequency of the rotor revolution is of the utmost importance. This frequency, often referred to as ' $1 \mathrm{P}$ ', may induce increased dynamic loads, e.g. due to rotor unbalances, wind shear and tower shadow. In addition, the higher 'P's' are of importance, e.g. the ' $2 \mathrm{P}$ ' and the ' $3 \mathrm{P}$ ', which are the frequencies of blades passing the tower on a two- and three-bladed turbine, respectively. When designing a turbine with variable speed, one must verify that the rotor speed of the turbine does not operate in or near the first natural frequency of the tower,

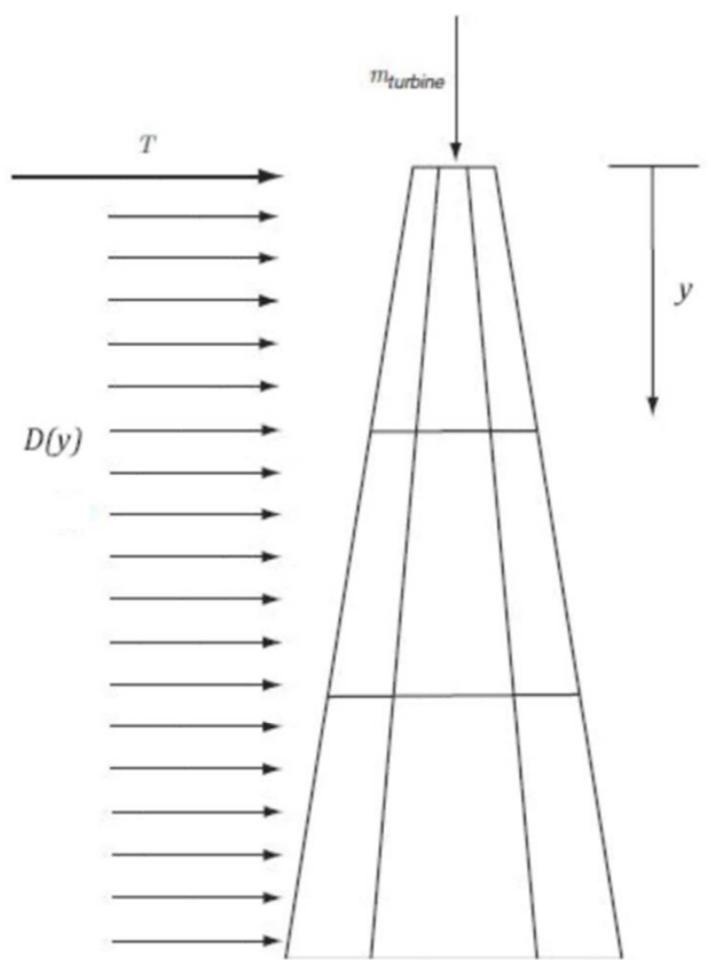

Figure 7. Loads on the Tower.

\section{Bending Stress}

For the horizontal drag applied by the wind on the tower, $(y)$ is defined per unit height as follows:

$$
D(y)=\frac{1}{2} \rho_{a} U^{2} C_{d} d_{A F}(y)
$$

For simplicity reasons, $U$ will be considered constant 
representing extreme wind speed. In order to find the stress due to the drag, the shear force $V$ acting in the tower under a distributed load $D(y)$ has to be calculated through the following integration:

$$
V=-\int D(y) d y
$$

Similarly, we integrate $V$ to obtain the bending moment $M(y)$

$$
M(y)=\int V(y) d y
$$

The maximum bending stress, $\sigma b, \max$, at any given height happens at a distance $x=d A F / 2$ from the centroid. And it is given by:

$$
\sigma_{b \max }=\frac{M(y) d_{A F}(y)}{2 I(y)}
$$

\section{Tabular Towers}

\subsection{Loads and Responses}

For the purpose of calculating section loads in the tower, the tower can be viewed as a cantilever beam as shown in Figure 8. External loads, figure 6, are applied at the tower top flange, which is located at a height $\mathrm{H}$ above the tower base. Note that this height may deviate somewhat from the hub height. Section loads in the tower at height $\mathrm{h}$ can be calculated from the loads applied at the top of the tower:

$$
\begin{gathered}
\mathrm{F}_{\mathrm{z}}(\mathrm{h})=\mathrm{F}_{\mathrm{zT}}+\rho_{\mathrm{t}} \int_{\mathrm{h}}^{\mathrm{H}} \mathrm{A}(\mathrm{z}) \mathrm{dz} \\
\mathrm{M}_{\mathrm{z}}(\mathrm{h})=\mathrm{M}_{\mathrm{zT}} \\
\mathrm{F}_{\mathrm{y}}(\mathrm{h})=\mathrm{F}_{\mathrm{yT}}+\mathrm{F}_{\mathrm{w}}(\mathrm{h})
\end{gathered}
$$

$$
\mathrm{M}_{\mathrm{x}}(\mathrm{h})=\mathrm{M}_{\mathrm{xT}}+\mathrm{F}_{\mathrm{yT}} *(\mathrm{H}-\mathrm{h})+\mathrm{M}_{\mathrm{w}}(\mathrm{h})+\mathrm{F}_{\mathrm{zT}} \cdot(\delta(\mathrm{H})-\delta(\mathrm{h}))
$$

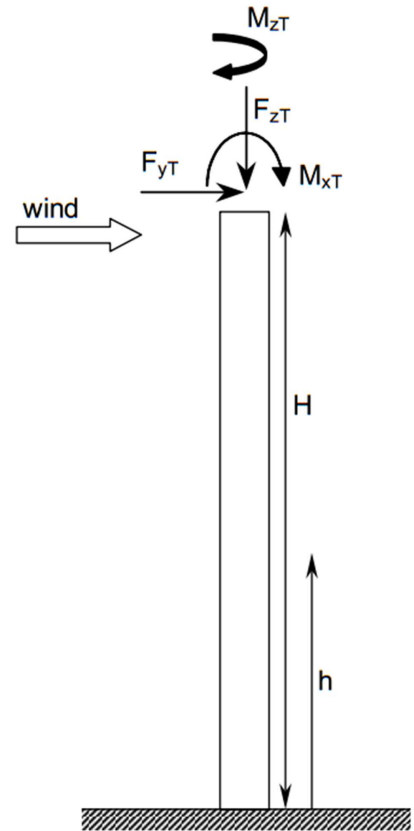

Figure 8. Cantilever beam model of a tubular tower.

$F_{y}=$ thrust from wind load

$M_{x}=$ bending moment from wind load

$F_{z}=$ gravity force

$M_{z}=$ torsional moment

$\rho_{t}=$ density of tower including appurtenances

$A(z)=$ cross sectional area as a function of height $\mathrm{z}$

$\delta=$ deflection of tower due to thrust from wind

External loads, here denoted by index T, are assumed to include the dynamic effect or gust factor (referring to a quasistatic approach). In $M_{x T}$, it is particularly important to include the contribution from the possible eccentricity of the nacelle. The section force $F_{w}(h)$ and the moment $M_{w}(h)$ from the wind load on the tower can be calculated as

$$
F_{w}(h)=1 / 2 \rho \int_{h}^{H} V(z)^{2} \varphi D(z) C(z) d
$$

$M_{w}(h)=1 / 2 \rho \int_{h}^{H}(H-h-z) V(z)^{2} \varphi D(z) C(z) d z$

Where

$\rho=$ air density

$V(z)=$ wind speed

$D(z)=$ outer tower diameter

$C(z)=$ form factor

$\varphi=$ gust factor

$C(z)$ depends on the Reynold's number, $R e=\frac{V D}{v}$ in which $v$ denotes the kinematic viscosity of air. At 20 $\mathrm{C}, v=15.09 * 10^{-6} \mathrm{~m}^{2} / \mathrm{sec}$. For painted steel towers $C(z)$ can be set to 0.6 .

\subsection{Extreme Loads}

For identification of the loads that govern the design, the specific combination of load components that produces the highest stress must be found. This can be quite a task when an aeroelastic analysis program is used that simulates a large number of load cases in 10-minute time series. Further, determination of which load case actually governs the design will most likely vary from different sections of the tower.

Alternatively, loads must be combined by taking the maximum of each load component from the particular load case where the most dominant load has its maximum, or more conservatively they can be combined by combining the maxima of the various load components regardless of which load case they actually appear in.

\subsection{Fatigue Loads}

Combining fatigue loads is a complicated task. When using the rain-flow method the load spectra for the different load components are normally not directly combinable. Therefore it might be a good idea, if possible, to combine the time series of the various load components resulting from the aero elastic simulation.

For example, the resulting bending moment in the direction $\theta$ relative to the y-axis can be calculated as 


$$
M_{\text {res }}=M_{x} \sin \theta+M_{y} \cos \theta
$$

in which $M_{x}$ and $M_{y}$ are the bending moments associated with the load components in the $\mathrm{x}$ and $\mathrm{y}$ directions, respectively. This could be taken even further to calculate the stress at relevant sections in the tower for every time step during the simulation and subsequently rain-flow count the resulting time series of the stress.

\subsection{Vortex Induced Vibrations}

The turbine must be checked for vortex induced vibration. The vortex excitation may occur during mounting of the turbine i.e. in a situation where the rotor and nacelle have not yet been mounted on the tower. DS410, according to which the critical wind speed $v_{r}$ can be calculated as

$$
v_{r}=\frac{n * D}{S t}
$$

Where

$n=$ tower natural frequency

$D=$ tower diameter

$S t=$ strouhal number

For conical towers, D should be set equal to the top diameter.

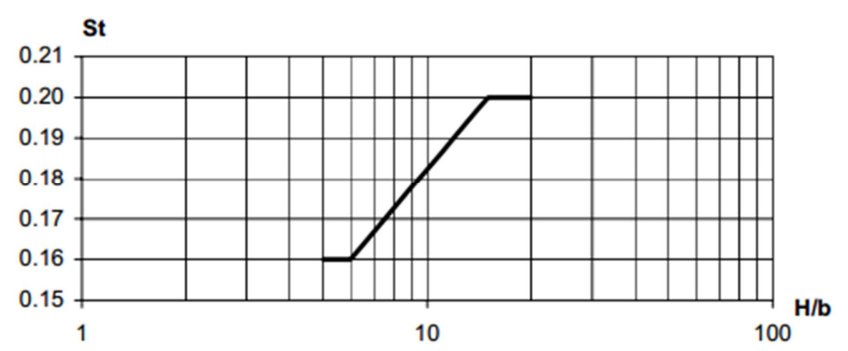

Figure 9. The Strouhal number vs. ratio between tower height $H$ and tower diameter $b$.

The analysis might prove that certain wind velocities should be avoided when erecting the tower. However, the sensitivity to vortex vibrations may be changed by temporary guy wiring of the tower or by mounting a temporary mass near the top of the tower. Normally, vortex-induced vibrations do not pose any problems after installation of the tower and the wind turbine. Once the nacelle is in place, its weight will lower the critical wind speed for vortex-induced vibrations to a low level typically below $10 \mathrm{~m} / \mathrm{s}$ which is within the interval of power production. When the blades rotate and pass the tower, they will reduce the wind speed and create turbulence in the wind that passes the tower behind the blades, thereby obstructing the generation of vortices.

Another aspect, which contributes to reducing the effect of vortex-induced vibrations, is the aerodynamic damping of the blades and the nacelle.

\subsection{Welded Joints}

Welds are, in general, treated in the same manner as the rest of the structure when a proper reduction factor for the weld quality and base material is included.
Table shows the recommended detail categories for bolts with rolled threads after heat treatment and common welds in tubular towers according to the standards Eurocode 3 and DS412. The given detail categories assume $100 \%$ controlled full penetration butt welds of quality level $\mathrm{B}$ according to DS/ISO 25817.

Table 3. Detail categories for bolts and common.

\begin{tabular}{ll}
\hline Weld & categories \\
\hline Plate to plate & 80 \\
Plate to flange & 71 \\
Plate to door frame & 80 \\
Axially loaded bolts & 71 \\
\hline
\end{tabular}

According to DNV (1987), the stress concentration factor for the single-sided plate tapering can be calculated as

$$
S C F_{\text {taper }}=1+6 \frac{e}{t_{2}\left(1+\left(\frac{t_{1}}{t_{2}}\right)^{2.5}\right)}
$$

in which $t_{1}$ and $t_{2}$ are the plate thickness of the lower and upper part of the tower shell, respectively, and the eccentricity e is given by

$$
e=1 / 2\left(t_{1}-t_{2}\right)
$$

\subsection{Stability Analysis}

The buckling strength of the tower usually governs the tower design as far as the shell thickness is concerned. The buckling strength of the tower can be analyzed using the approach described in Annex D of DS449 combined with DS412, DIN 18800 or other recognized standards. In the following, the method suggested in the Danish standard is presented. Stresses owing to the axial force, $\sigma_{a d}$, and owing to the bending moment, $\sigma_{b d}$, are given by

$$
\begin{gathered}
\sigma_{a d}=\frac{N_{d}}{2 \pi R t} \\
\sigma_{b d}=\frac{M_{d}}{\pi R^{2} t}
\end{gathered}
$$

A reduction factor $\varepsilon$ is calculated as

$$
\begin{gathered}
\varepsilon_{\mathrm{a}}=\frac{0.83}{\sqrt{1+0.01 \frac{\mathrm{R}}{\mathrm{t}}}} \\
\varepsilon_{\mathrm{b}}=0.1887+0.8113 \varepsilon_{\mathrm{a}} \\
\varepsilon=\frac{\varepsilon_{\mathrm{a}} \sigma_{\mathrm{ad}}+\varepsilon_{\mathrm{b}} \sigma_{\mathrm{bd}}}{\sigma_{\mathrm{ad}}+\sigma_{\mathrm{bd}}}
\end{gathered}
$$

According to theory of elasticity, the critical compressive stress is

$$
\sigma_{e l}=\frac{E_{d}}{\frac{R}{t} \sqrt{3(1-v)^{2}}}
$$

The relative slenderness ratio for local buckling is

$$
\lambda_{a}=\sqrt{\frac{f_{y d}}{\varepsilon \sigma_{e l}}}
$$

If $\lambda_{a} \leq 0.3$ the critical compressive stress $\sigma_{c r}$ is given by 


$$
\sigma_{c r}=f_{y d}
$$

If $0.3<\lambda_{a} \leq 1$ the critical compressive stress $\sigma_{c r}$ is given by

$$
\sigma_{c r}=\left(1.5-0.913 \sqrt{\lambda_{a}}\right) f_{y d}
$$

However if the tower height $\mathrm{H}$ does not exceed $1.42 R \sqrt{R / t}$ then

$$
\sigma_{c r}=f_{y d}
$$

From theory of elasticity, the Euler force for a cantilever beam is given by

$$
N_{e l}=\frac{\frac{1}{4} \pi^{2} E_{d} \pi R^{3} t}{H^{2}}
$$

The relative slenderness ratio for global stability is

$$
\lambda_{r}=\sqrt{\frac{\sigma_{c r}}{\left(\frac{N_{e l}}{2 \pi R t}\right)}}
$$

The core radius $\mathrm{k}$ of a tube is given by

$$
k=\frac{R}{2}
$$

For cold-formed welded towers, the equivalent geometrical imperfection can now be calculated as

$$
e=0.49\left(\lambda_{r}-0.2\right) k
$$

For welded towers it can be calculated as

$$
e=0.34\left(\lambda_{r}-0.2\right) k
$$

However if $\lambda_{r} \leq 0.2$ then $e=0$

If $e>\frac{2}{1000} \mathrm{H}$ then an additional increment $\Delta e=(e-$ $\frac{2}{1000} \mathrm{H}$ ) is to be added to e.

Finally, the following inequality must be fulfilled

$$
\frac{N_{d}}{2 \pi R t}+\frac{N_{e l}}{N_{e l}-N_{d}} * \frac{M_{d}+N_{d} e}{\pi R^{2} t}<\sigma_{c r}
$$

\begin{tabular}{|c|c|c|c|c|c|c|c|}
\hline Material: & \multicolumn{4}{|c|}{ Steel } & \multicolumn{3}{|c|}{ Concrete } \\
\hline $\begin{array}{l}\text { Wind turbine: } \\
\text { WKA-60-II } \\
\text { Rotor: } \\
3 \text { blades, } \varnothing 60 \mathrm{~m} \\
\text { Rotor speed: } \\
\mathrm{n}=23 \mathrm{rpm}= \\
0.3833 \mathrm{~Hz} \\
\text { Blade frequency: } \\
3 \times \mathrm{n}=1.15 \mathrm{~Hz} \\
\text { Head mass: } 207 \mathrm{t} \\
\text { hub height: } 50 \mathrm{~m}\end{array}$ & $\underset{\begin{array}{c}\text { Cylindri- } \\
\text { cal tower }\end{array}}{ }$ & Conical & $\underset{\substack{\text { Lattice } \\
\text { tower }}}{|||| \mid}$ & $\begin{array}{l}\text { Guyed lattice } \\
\text { tower }\end{array}$ & Pre-fabricated & $\prod_{\substack{\text { on-site } \\
\text { concrete }}}$ & $\underbrace{}_{\substack{\text { on-site con- } \\
\text { crete, conical }}}$ \\
\hline $\begin{array}{l}1^{\text {st }} \text { tower natural bending } \\
\text { frequency } \mathrm{f}_{0.1}[\mathrm{~Hz}] \\
\text { Frequency ratio } \mathrm{f}_{0.1} / \mathrm{n}[\mathrm{p}]\end{array}$ & $\begin{array}{l}0.55 \\
1.44\end{array}$ & $\begin{array}{l}0.56 \\
1.46\end{array}$ & $\begin{array}{l}0.55 \\
1.44\end{array}$ & $\begin{array}{l}0.55 \\
1.44\end{array}$ & $\begin{array}{l}0.65 \\
1.70\end{array}$ & $\begin{array}{l}0.96 \\
2.50\end{array}$ & $\begin{array}{l}0.96 \\
2.50\end{array}$ \\
\hline $\begin{array}{l}\varnothing_{\text {top }} \quad[\mathrm{m}] \\
\varnothing \text { bottom }[\mathrm{m}] \\
\text { Wall thickness [mm] }\end{array}$ & $\begin{array}{l}3.50 \\
3.50 \\
\text { stepped } \\
35-20\end{array}$ & $\begin{array}{l}3.20 \\
7.50 \\
20\end{array}$ & $\begin{array}{l}3.10 \\
4.30 \\
20\end{array}$ & $\begin{array}{l}2.70 \\
2.70 \\
20\end{array}$ & $\begin{array}{l}3.50 \\
3.50 \\
\text { stepped } \\
520 / 250\end{array}$ & $\begin{array}{l}3.30 \\
5.40 \\
300\end{array}$ & $\begin{array}{l}3.50 \\
8.10 \\
300\end{array}$ \\
\hline Tower mass $\quad[t]$ & 114 & 90 & 87 & $\begin{array}{l}63+\text { guy } \\
\text { wires }\end{array}$ & 430 & 455 & 540 \\
\hline $\begin{array}{l}\text { Relative costs for } \\
\text { supporting structure } \\
{[\%]}\end{array}$ & 230 & 185 & 175 & 200 & 100 & $\begin{array}{l}\text { Not pre- } \\
\text { stressed: } 115 \\
\text { Prestressed: } \\
160\end{array}$ & $\begin{array}{l}\text { Not pre- } \\
\text { stressed: } 135 \\
\text { prestressed: } \\
185\end{array}$ \\
\hline
\end{tabular}

$N_{d}=$ design axial force

$M_{d}=$ designed bending moment

$R=$ tower radius

$t=$ tower shell thickness

$\mathrm{H}=$ tower height

$E_{d}=$ designed modulus of elasticity

$\mathrm{V}=$ Poisson's ratio

$f_{y d}=$ designed yield stress

Figure 10. Tower material comparison between steel and concrete.

\section{Modeling of Tower}

\subsection{Basic Assumption}

1. The basic structural model of the tower is represented by an equivalent long, slender cantilever beam built from segments (modules) having different but uniform cross-sectional properties.

2. The tower is cantilevered to the ground, and is carrying a concentrated mass at its free end approximating the inertia properties of the nacelle/rotor unit. This mass is assumed to be rigidly attached to the tower top.

3. Material of construction is linearly elastic, isotropic and homogeneous. The tower has a thin-walled circular cross section.

4. The Euler-Bernoulli beam theory is used for predicting directions. Secondary effects such as axial and shear deformations, and rotary inertia are neglected.

5. Distributed aerodynamic loads are restricted to profile 
drag forces. A two-dimensional (2D) steady flow model is assumed.

6. Nonstructural mass will not be optimized in the design process. Its distribution along the tower height will be taken equal to some fraction of the structural mass distribution.

7. Structural analysis is confined only to the case of flapping motion (i.e. bending perpendicular to the plane of rotor disk).

\subsection{Design Requirements of a Tower}

\subsubsection{Light Weight Design}

A minimum weight structural design is of paramount importance for successful and economic operation of a wind turbine. The reduction in structural weight is advantageous from the production and cost points of view. For a tower composed of Ns segments the weight (mass) function, to be minimized, can be expressed in the non-dimensional form minimize

$$
M=\sum_{k=1}^{N_{s}} D_{k} t_{k} H_{k}
$$

Where $\mathrm{D}=$ diameter= thickness and $\mathrm{H}=$ height of tower

\subsubsection{High Stiffness}

The main tower structure must possess an adequate stiffness level. Maximization of the stiffness is essential to enhance the overall structural stability and decrease the possibility of fatigue failure. For a cantilevered tower, stiffness can reasonably be measured by the magnitude of a horizontal force applied at the free end and producing a maximum direction of unity maximize

$$
S=1 / \sum_{k=1}^{N_{S}} \frac{H_{k}}{I_{k}}\left[1-\left(x_{k+1}+x_{k}+\frac{1}{3}\left(x_{k+1}^{2}+x_{k} x_{k+1}+x_{k}^{2}\right)\right.\right.
$$

\subsubsection{High (Stiffness/Mass) -Ratio}

Maximization of the stiffness-to-mass ratio is directly related to the physical realities of the design, is a better and more straight forward design criterion than maximization of the stiffness alone or minimization of the structural mass alone.
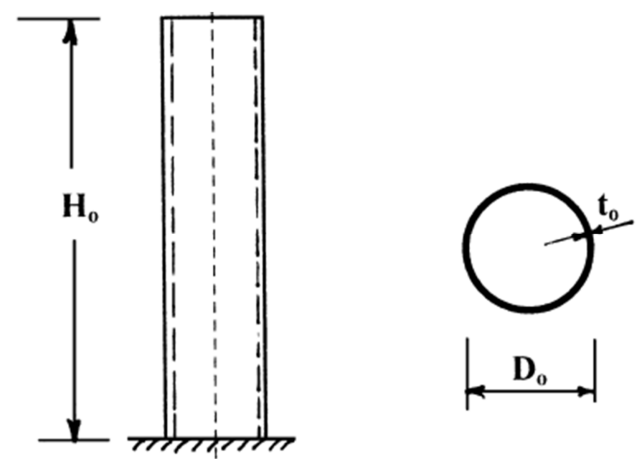

Figure 11. Thin walled tabular tower configuration.

\subsubsection{Design for Minimum Vibration}

Minimization of the overall vibration level is one of the most cost-effective solutions for a successful wind turbine design. It fosters other important design goals, such as long fatigue life, high stability and low noise level.

Frequency-placement criterion. Reduction of vibration can be achieved by separating the natural frequencies of the structure from the exciting frequencies to avoid large amplitudes caused by resonance.

\section{Result and Discussion}

\subsection{Bending Stress Analysis as Simple Cantilever Beam}

Considering the force applied on a tower is the trust force, atmospheric pressure and its own weight the following table shows the reaction forces and maximum bending moment which occur in matlab 2018 code result and it is based on considering the load as uniformly (2) and point load (1).

$\mathrm{a}=$ length of beam $(\mathrm{m})$

$\mathrm{b}=$ load type

$\mathrm{c}=$ applied load $(\mathrm{KN})$

$\mathrm{d}=$ length of uniformly distributed load (m)

$\mathrm{e}=$ center of gravity from left end (m)

$\mathrm{f}=$ left support reaction $(\mathrm{KN})$

$\mathrm{g}=$ right support reaction $(\mathrm{KN})$

$\mathrm{h}=$ maximum bending moment

Atmospheric + thrust force $=456 \mathrm{KN}$

Gravity force due to weight $=1277.3 \mathrm{KN}$

Thrust force $=354.9 \mathrm{KN}$

\begin{tabular}{|c|c|c|c|c|c|c|c|c|}
\hline & $\mathbf{a}$ & b & c & d & $\mathbf{e}$ & $\mathbf{f}$ & $\mathbf{g}$ & h \\
\hline 1 & 80 & 2 & 456 & 80 & 20 & 27360 & 9120 & 273600 \\
\hline 2 & 80 & 2 & 456 & 80 & 40 & 18240 & 18240 & 364800 \\
\hline 3 & 80 & 2 & 456 & 80 & 60 & 9120 & 27360 & 273600 \\
\hline 4 & 80 & 2 & 1277.3 & 80 & 20 & 76638 & 25546 & 766380 \\
\hline 5 & 80 & 2 & 1277.3 & 80 & 40 & 51092 & 51092 & 1021840 \\
\hline 6 & 80 & 2 & 1277.3 & 80 & 60 & 25546 & 76638 & 766380 \\
\hline 7 & 80 & 1 & 354.9 & 20 & 20 & 266.175 & 88.725 & 5323.5 \\
\hline 8 & 80 & 1 & 354.9 & 80 & 40 & 177.45 & 177.45 & 7098 \\
\hline 9 & 80 & 1 & 354.9 & 80 & 60 & 88.725 & 266.175 & 5323.5 \\
\hline
\end{tabular}

Table 4. Matlab outputs for reaction forces and bending moment. 


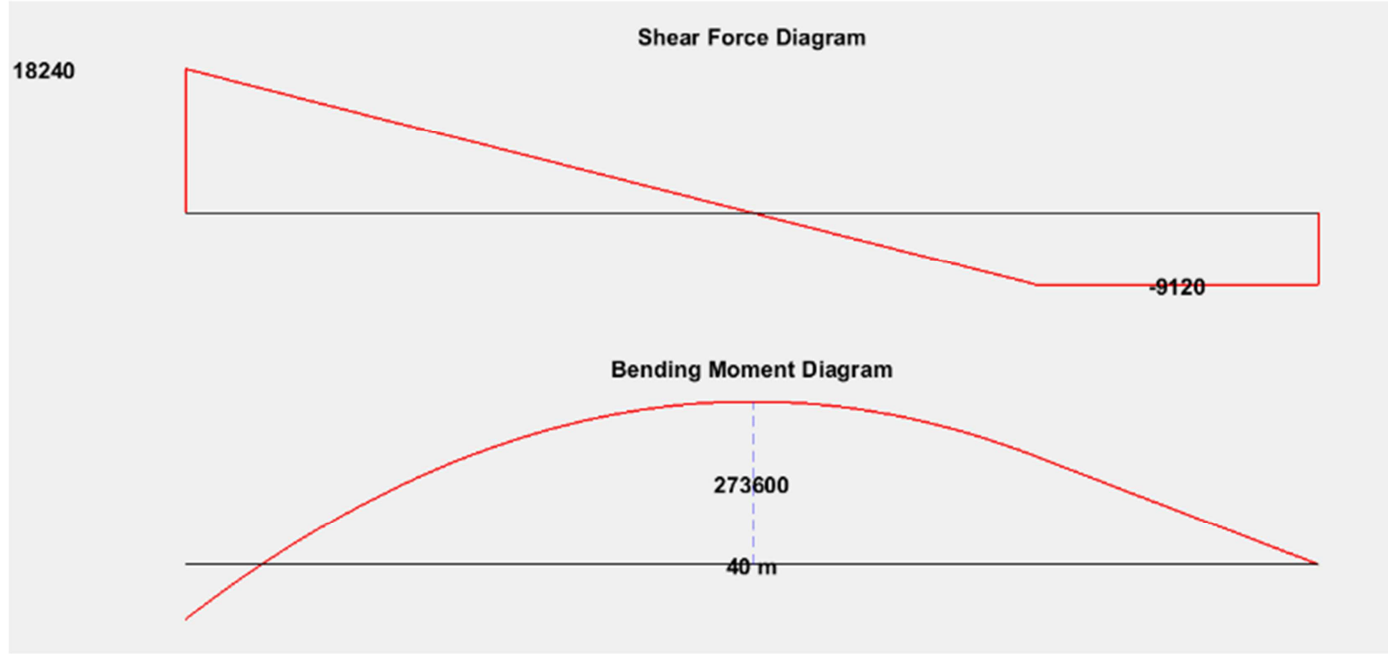

Figure 12. Force applied on a tower as the trust force at $l=20$.

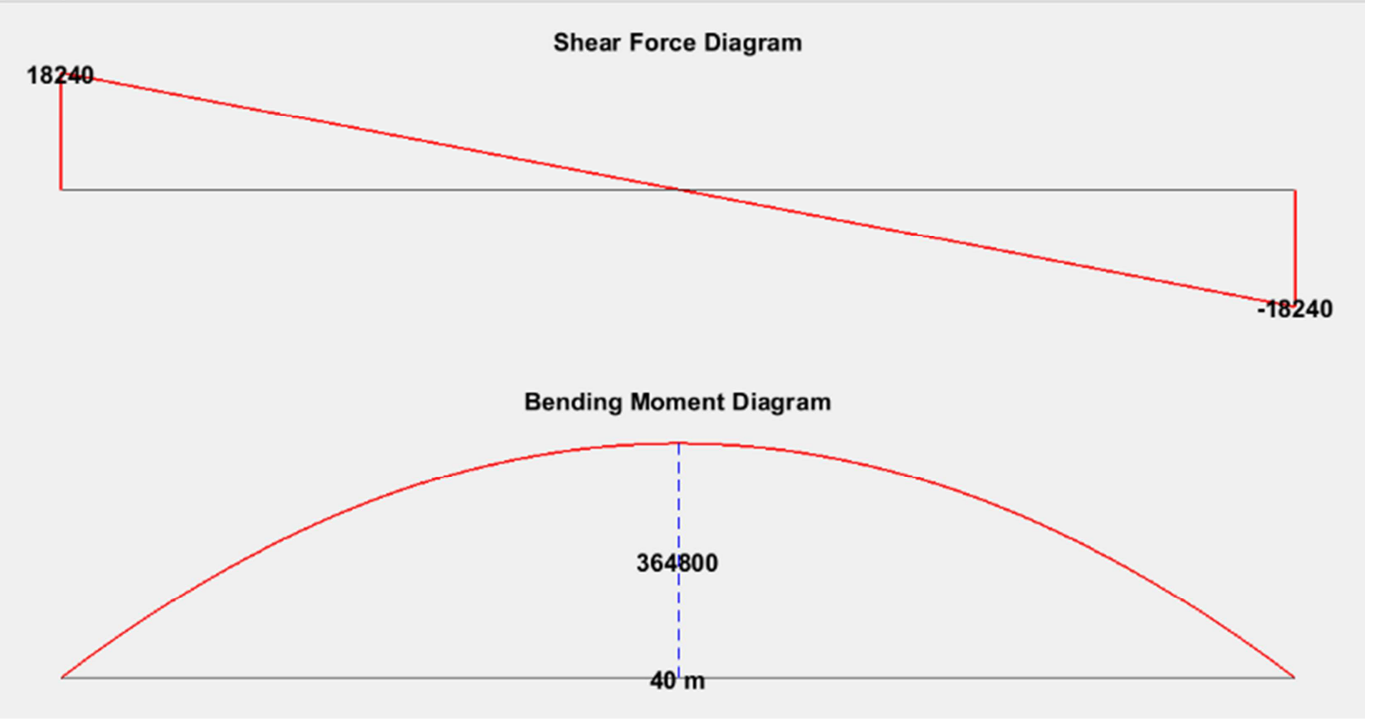

Figure 13. Matlab shear force and bending diagram at $l=40$.
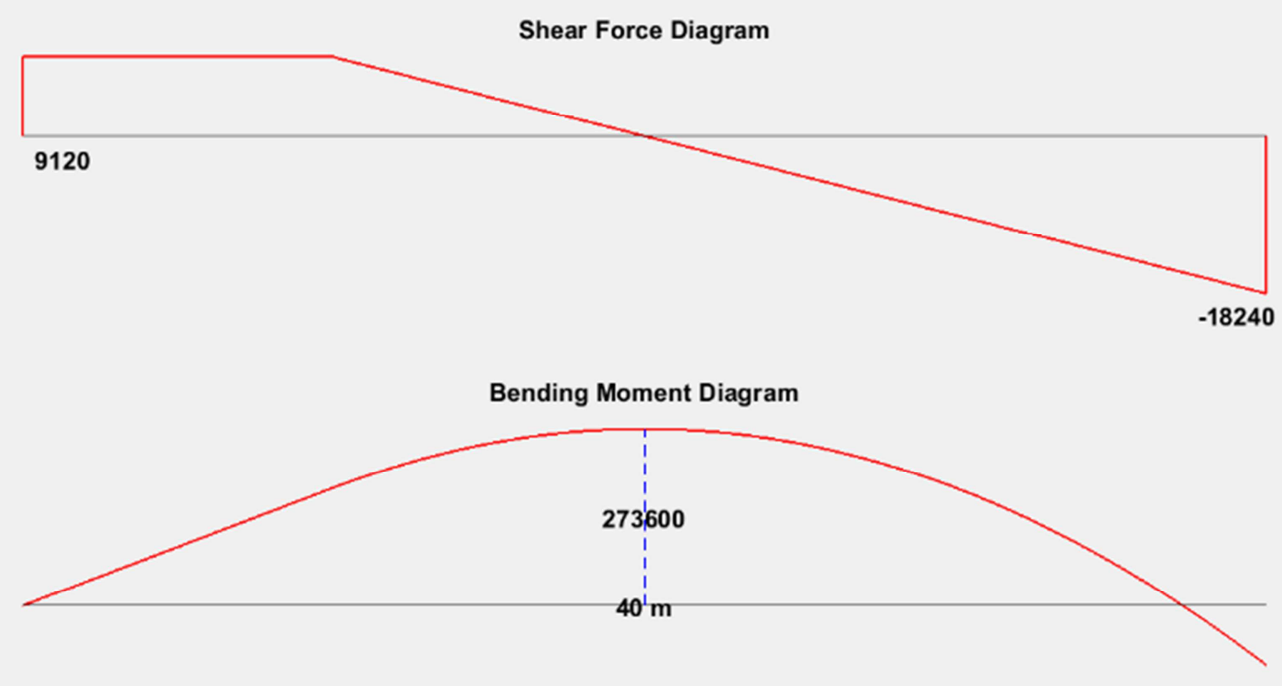

Figure 14. Matlab shear force and bending diagram at $l=60$.

Due to its own weight 


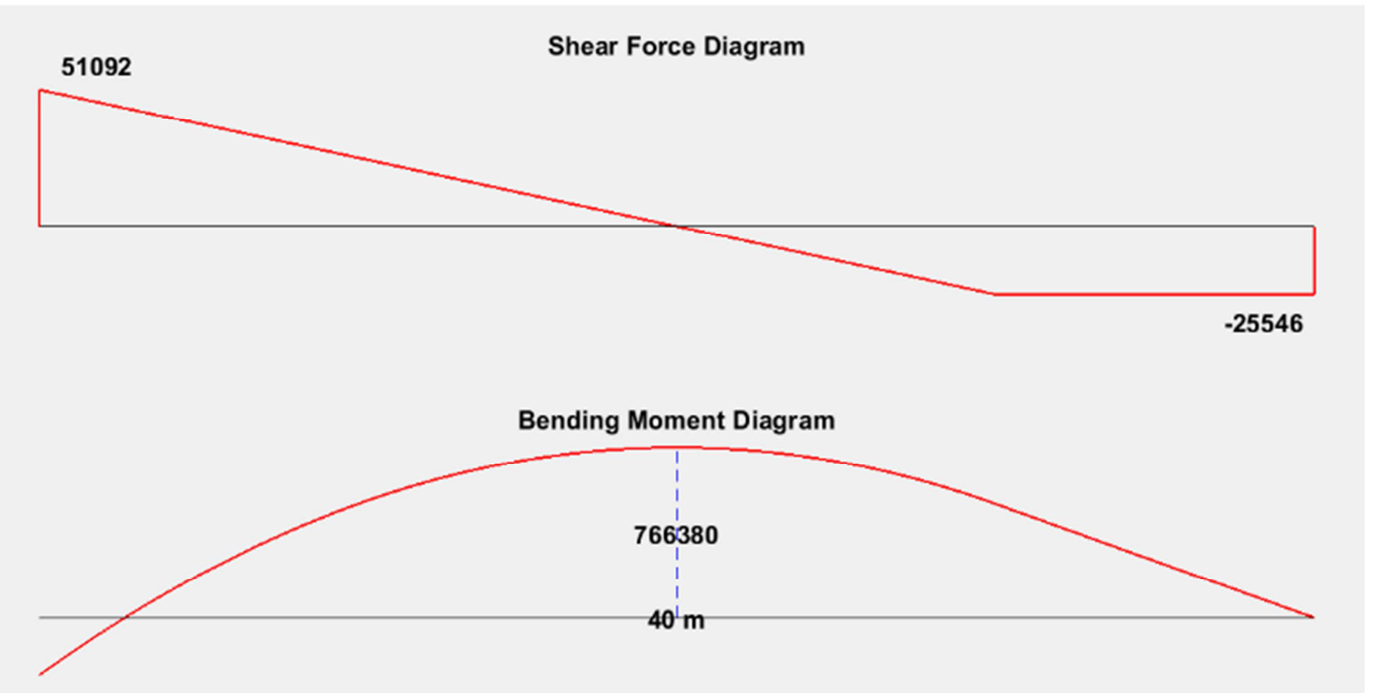

Figure 15. Matlab output of tower due to own weight at $l=20$.
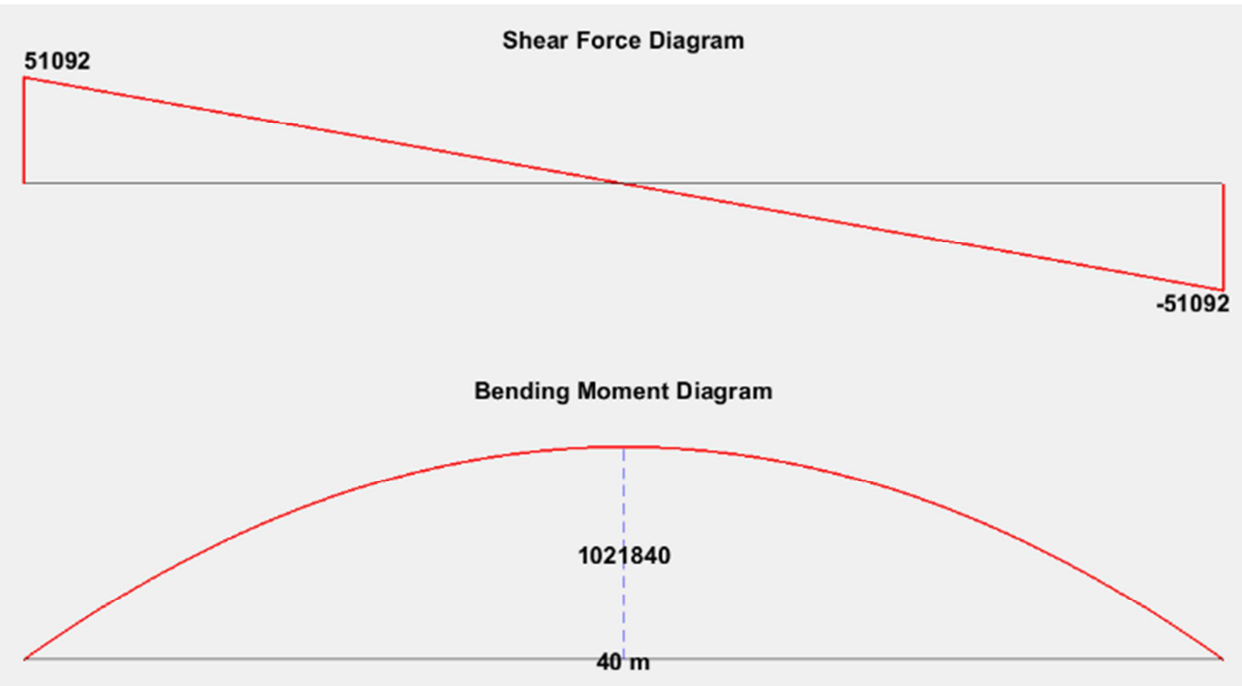

Figure 16. Matlab output of tower due to own weight at $l=40$.

Shear Force Diagram
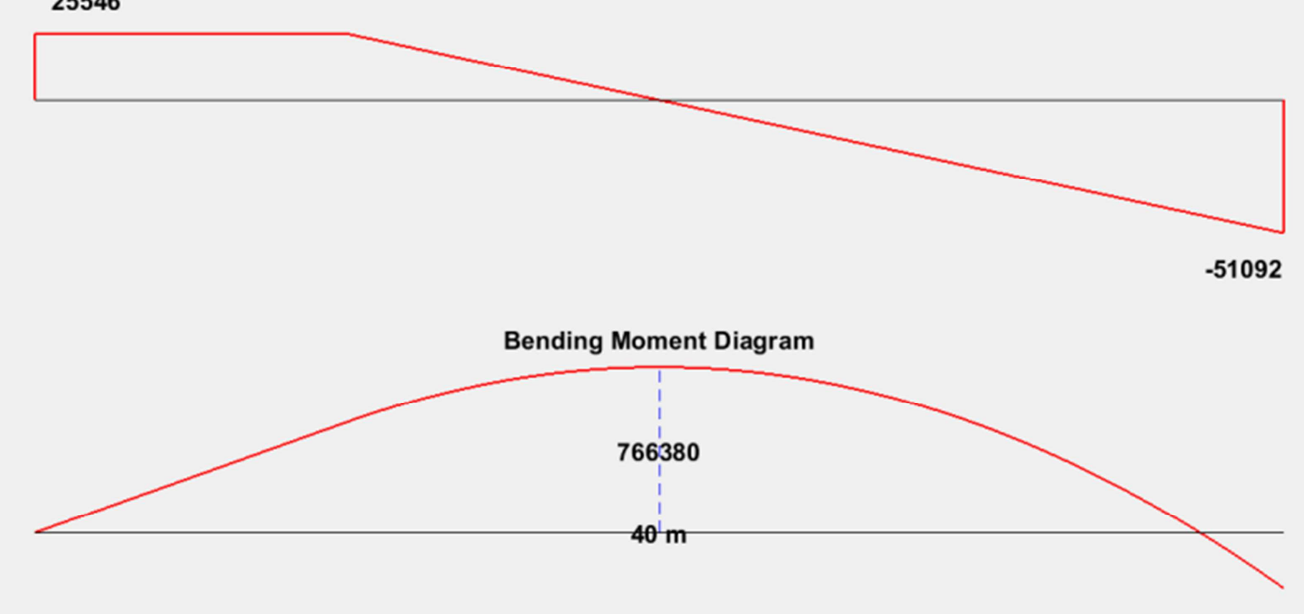

Figure 17. Matlab output of tower due to own weight at $l=60$

Due to atmospheric pressure 


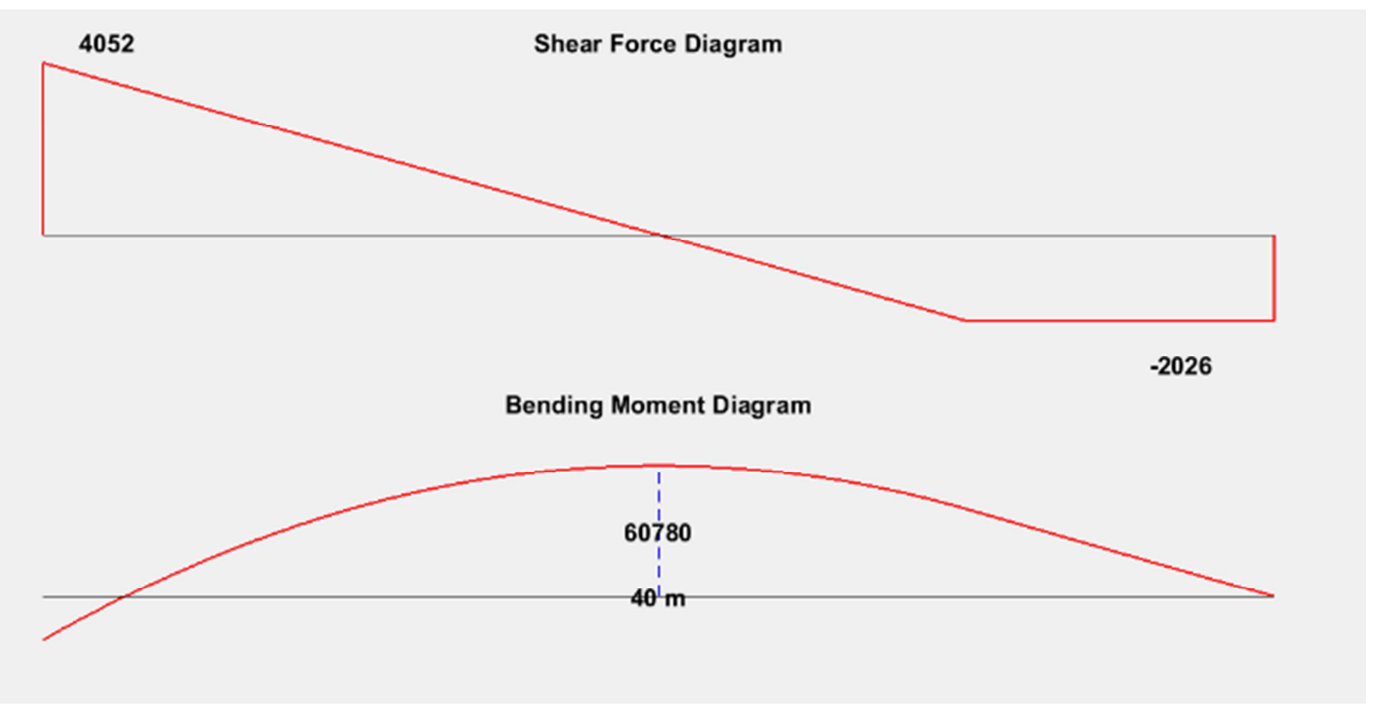

Figure 18. Matlab output due to atmospheric pressure at $l=20$.

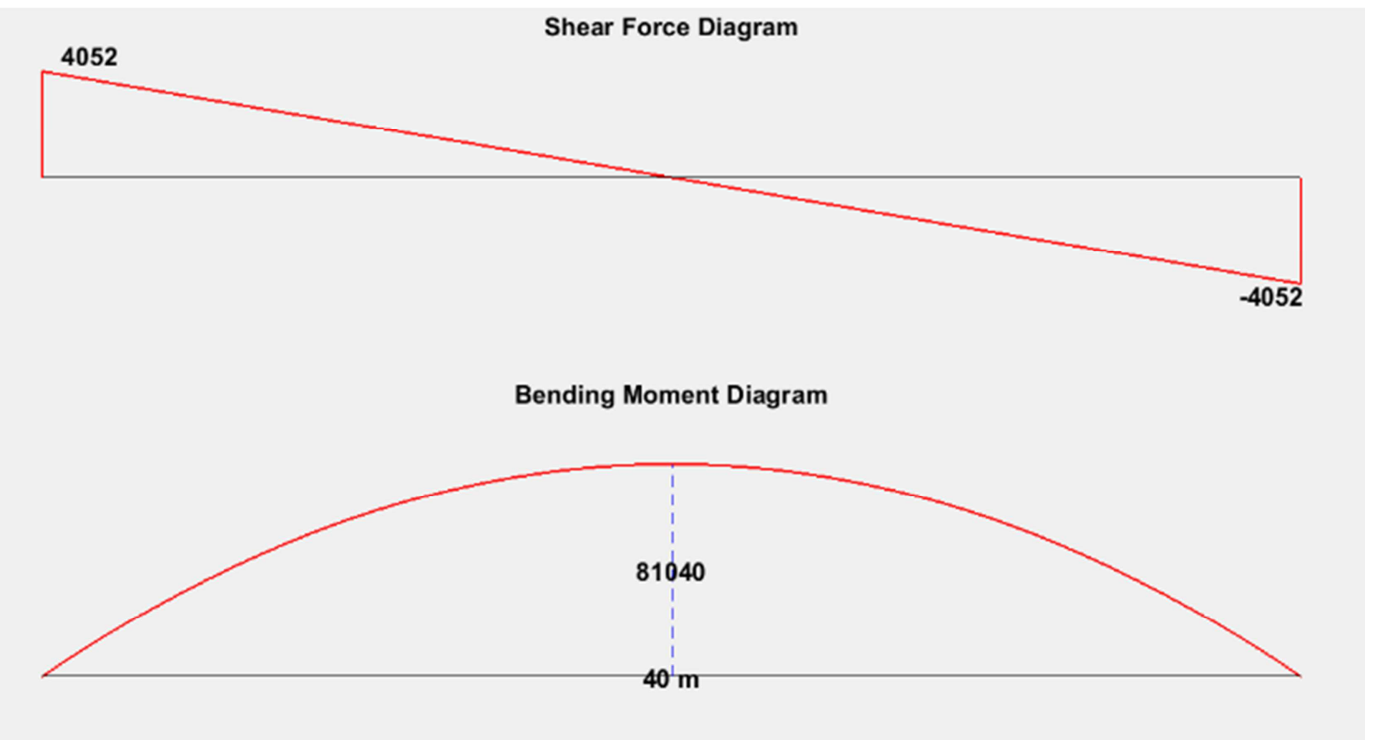

Figure 19. Matlab output due to atmospheric pressure at $l=40$.

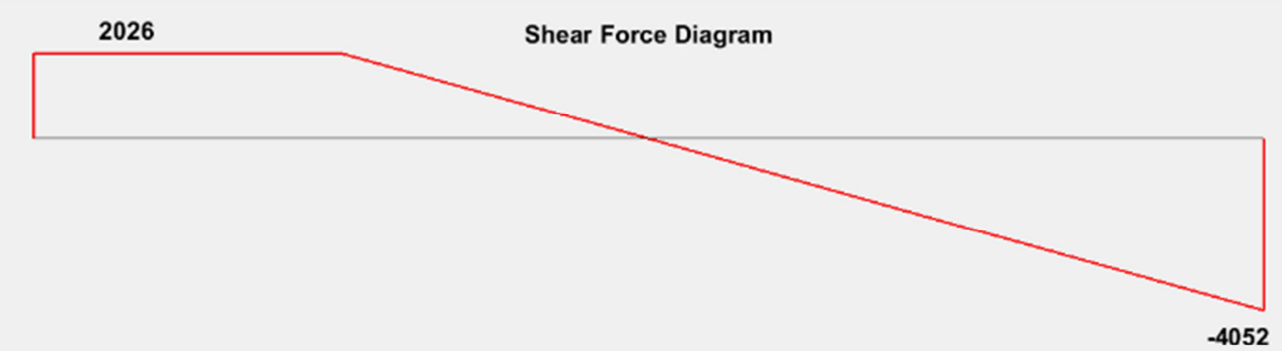

Bending Moment Diagram

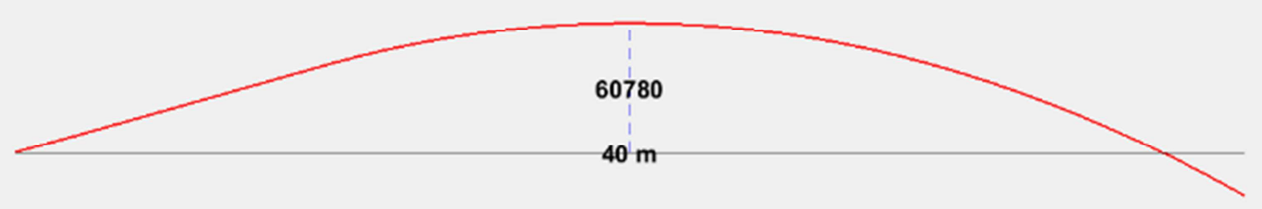

Figure 20. Matlab output due to atmospheric pressure at $l=60$. 
Considering the trust force as point load

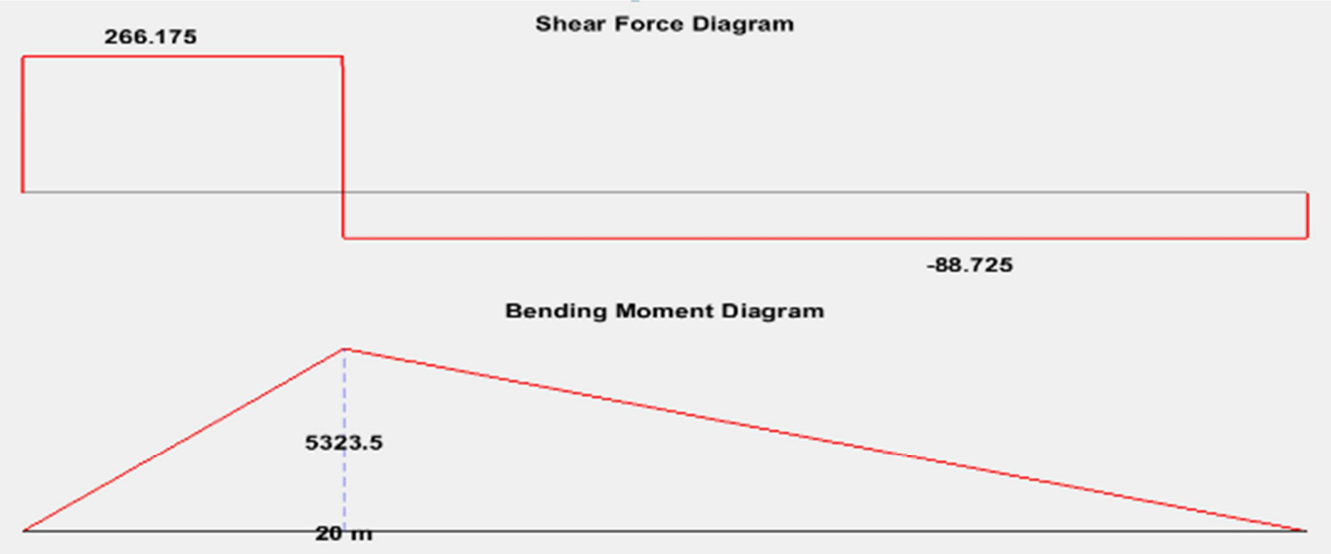

Figure 21. Matlab output as point load $l=20$.

177.45

Shear Force Diagram

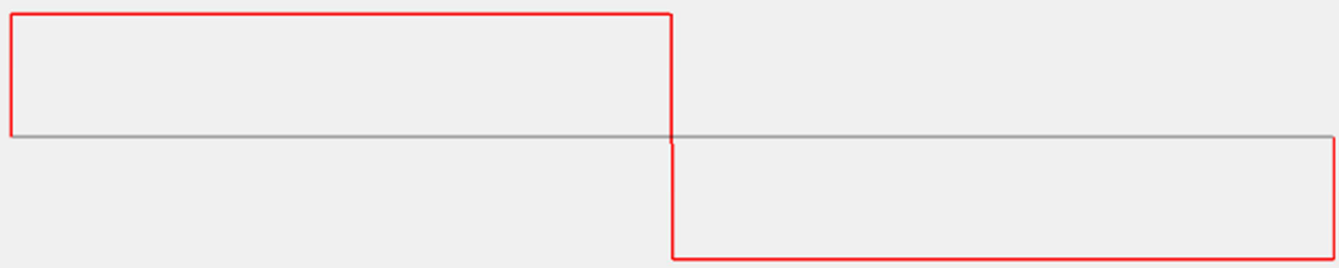

$-177.45$

Bending Moment Diagram

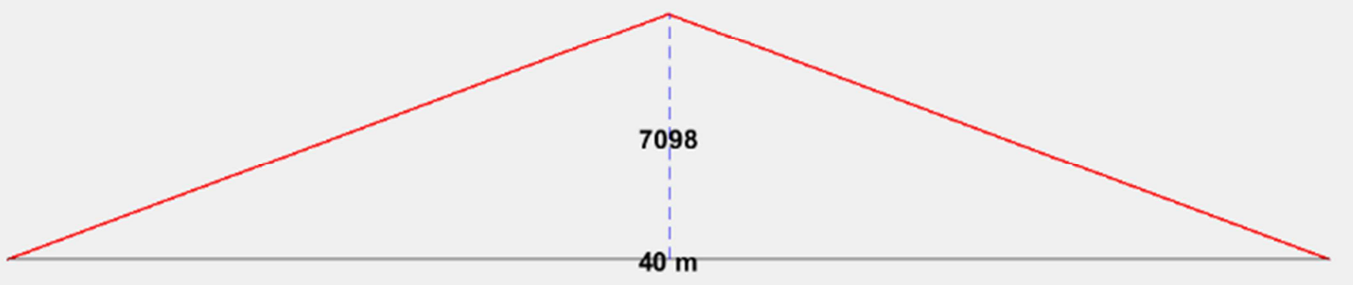

Figure 22. Matlab output as point load $l=40$.

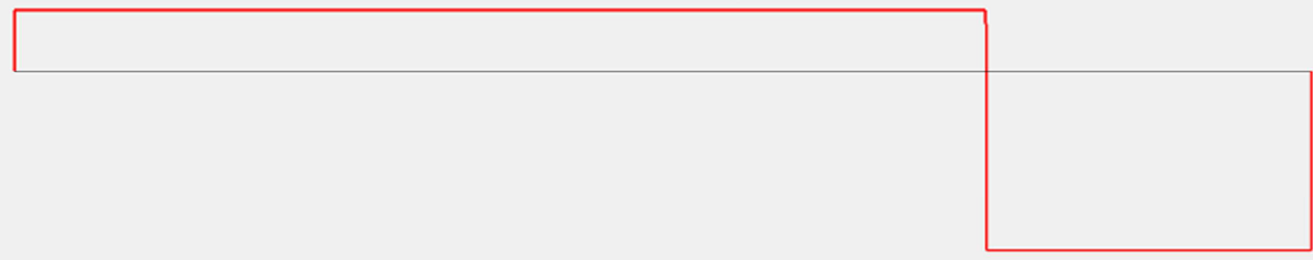

$-266.175$

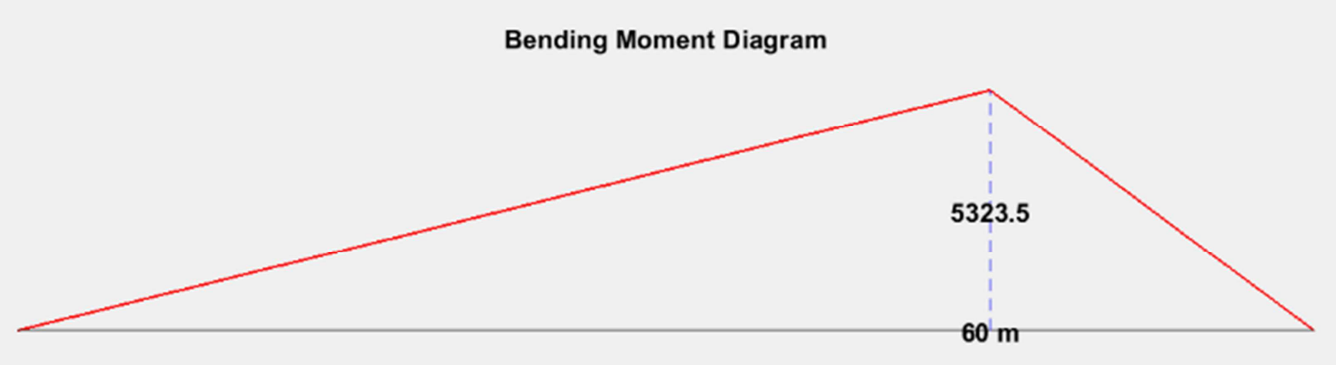

Figure 23. Matlab output as point load $l=60$. 
Vibrational analysis

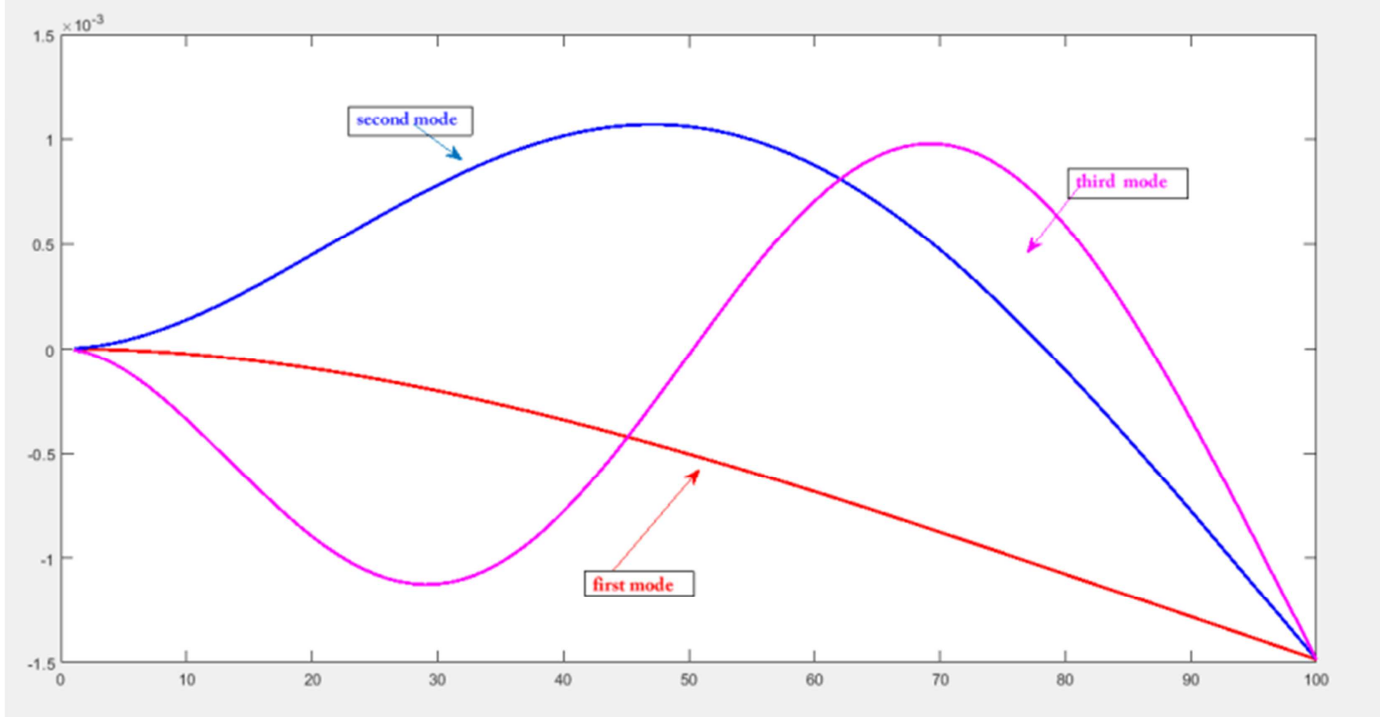

Figure 24. Vibrational analysis for different modes of frequency.

Frequency $=$

$\mathrm{fl}=0.3739 \mathrm{~Hz}$

Natural frequency of tower

Table 5. The first three natural frequencies ( $\mathrm{rad} / \mathrm{sec})$.

\begin{tabular}{lll}
\hline Theory & Fem & Error \% \\
\hline 1.0139 & 1.0127 & -0.1133 \\
6.3368 & 6.3468 & 0.1568 \\
17.7719 & 17.7711 & -0.0045 \\
\hline
\end{tabular}

Table 6. Euler buckling load (N).

\begin{tabular}{lll}
\hline Theory & Fem & Error \% \\
\hline $1.0 \mathrm{e}+09 * 1.6751$ & 0.0000 & -0.0000 \\
\hline
\end{tabular}

Mode shape as abeam ( $\mathrm{rad} / \mathrm{sec})$

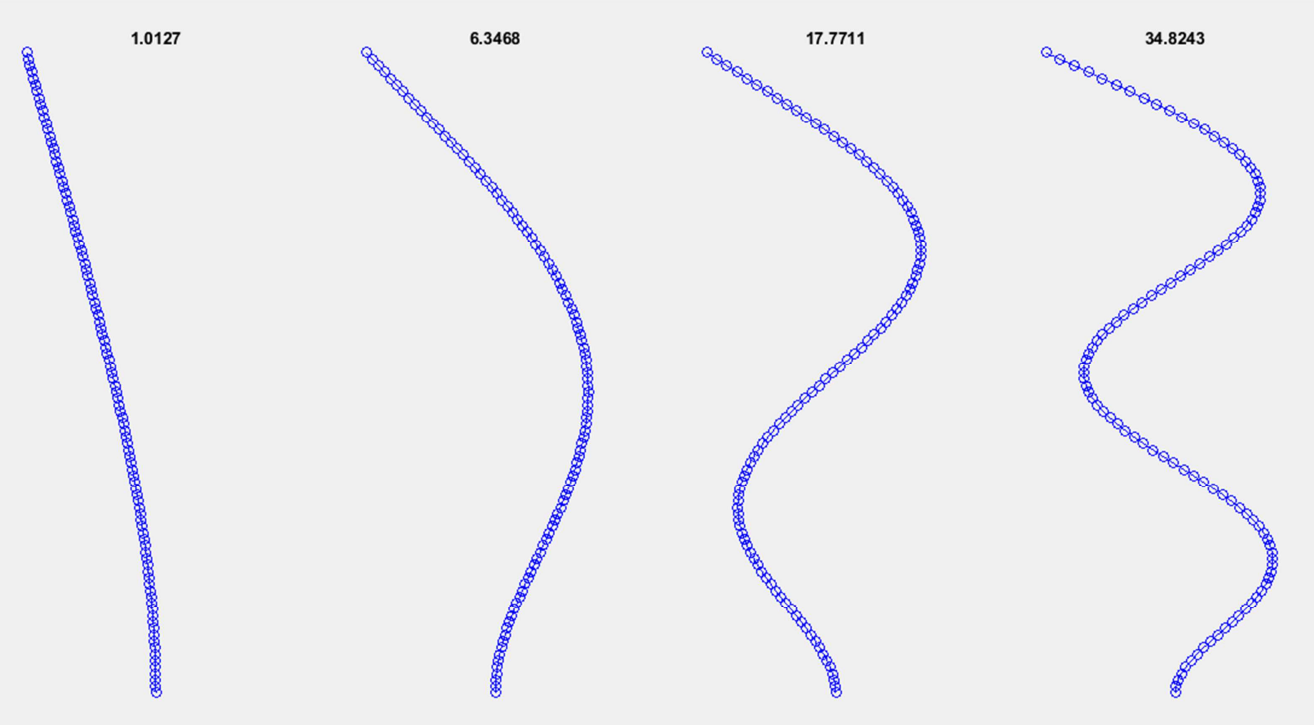

Figure 25. Mode shape as abeam ( $\mathrm{rad} / \mathrm{sec})$.

Buckling mode 


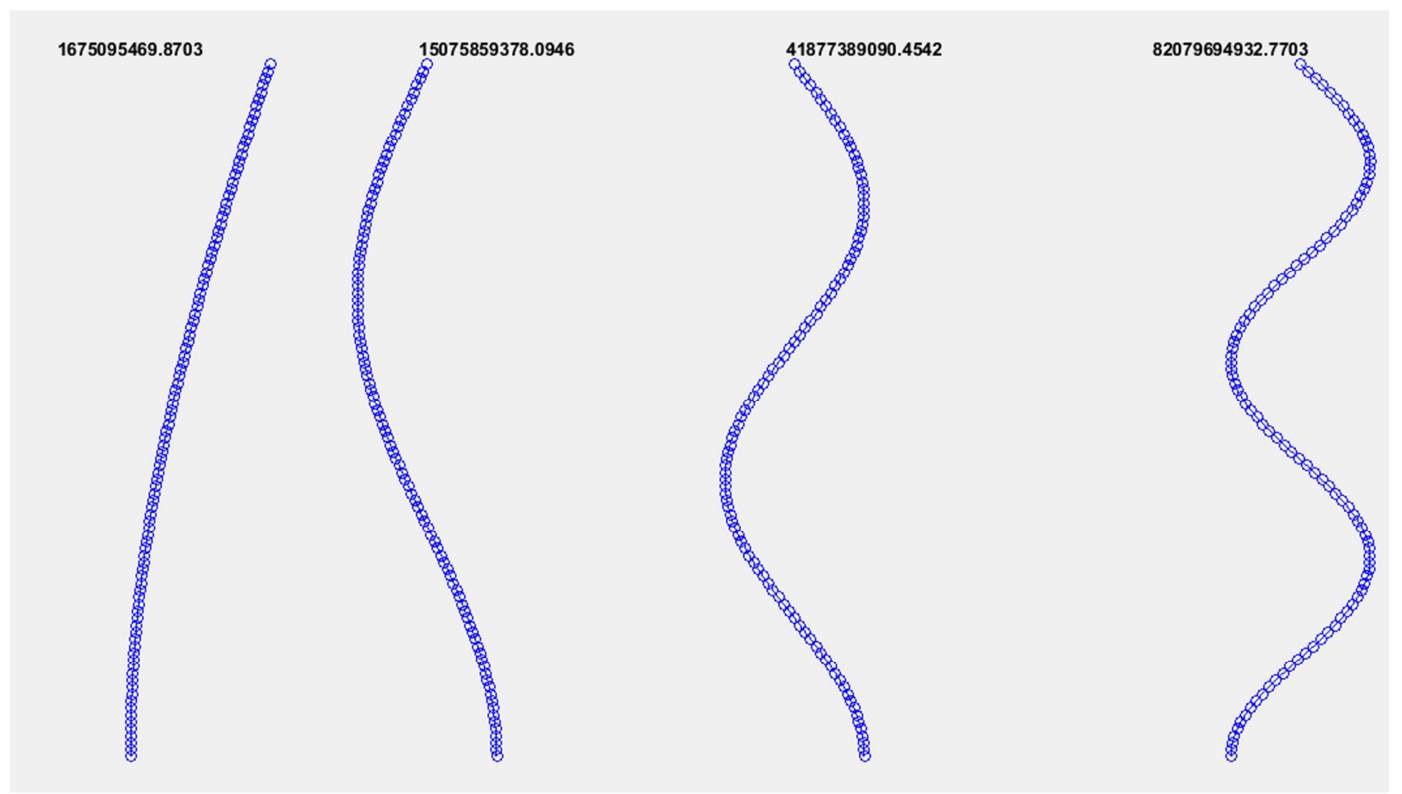

Figure 26. Matlab output for buckling analysis.

\subsection{ANSYS ${ }^{\circledR} 19.2$ Analysis of Tower}

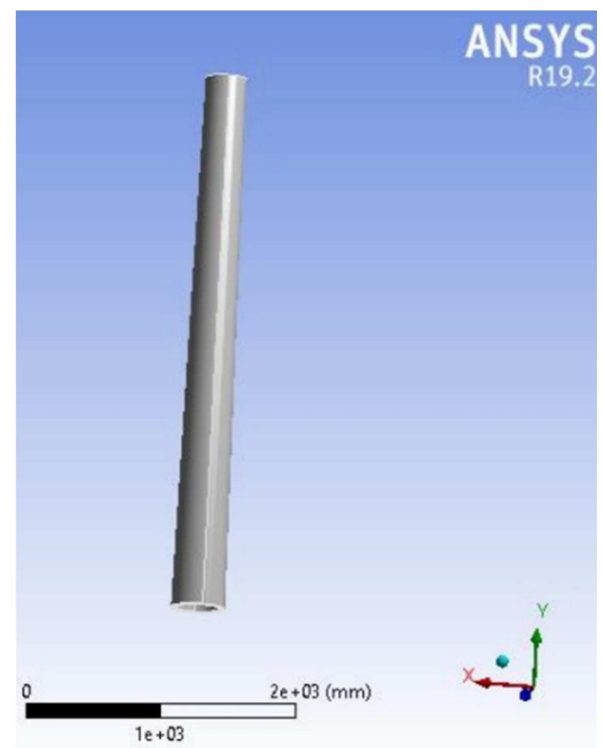

Figure 27. Tower model imported in ANSYS workbench.

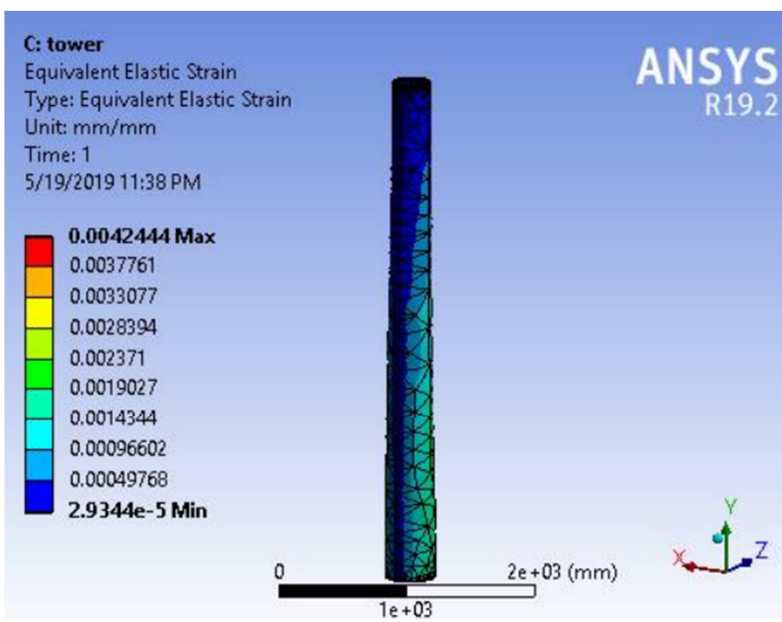

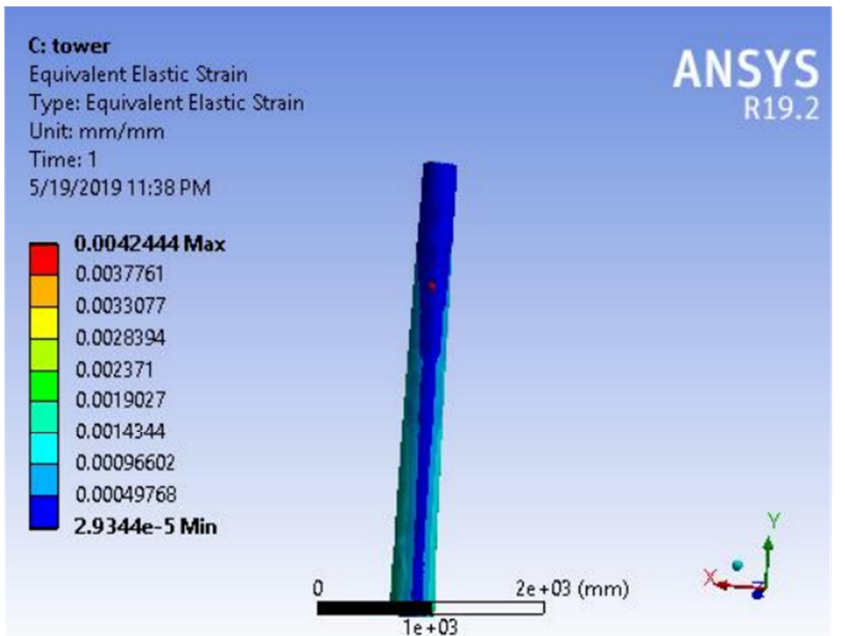

Figure 28. Tower equivalent strain.

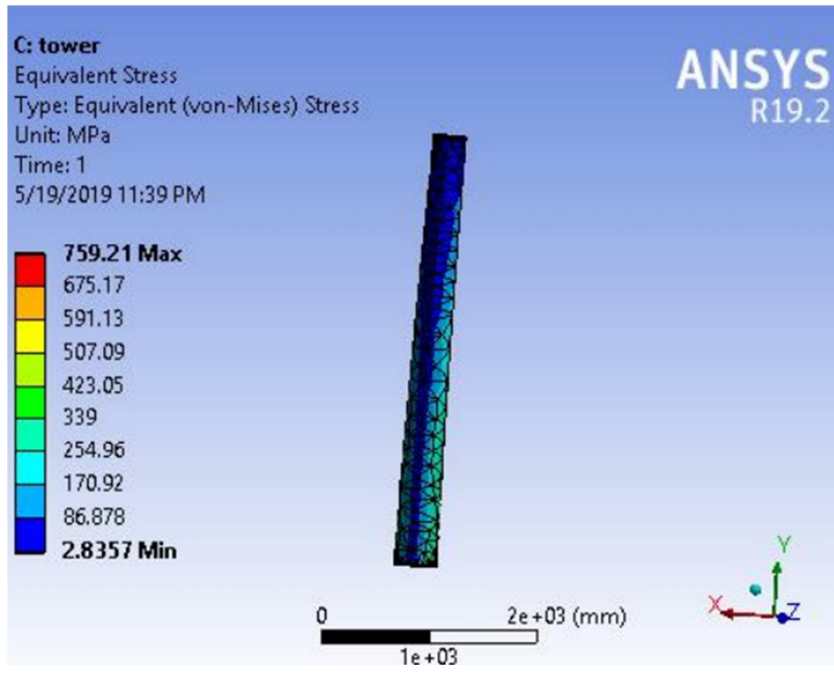




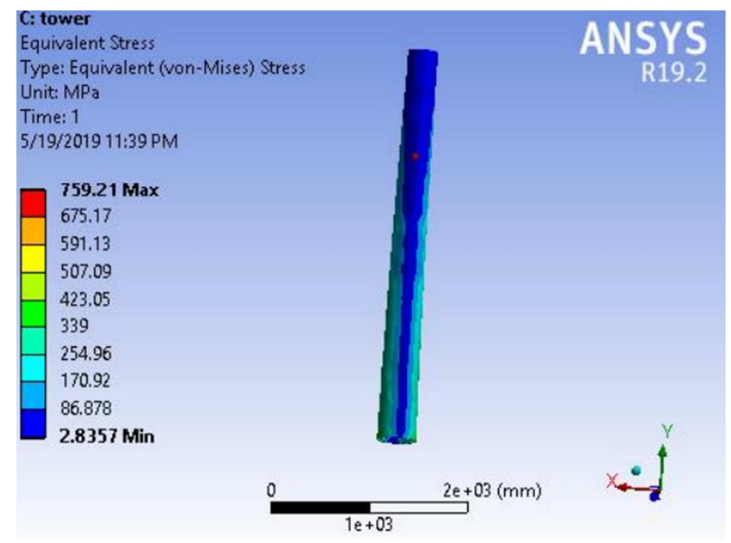

Figure 29. Tower von-misses stress.
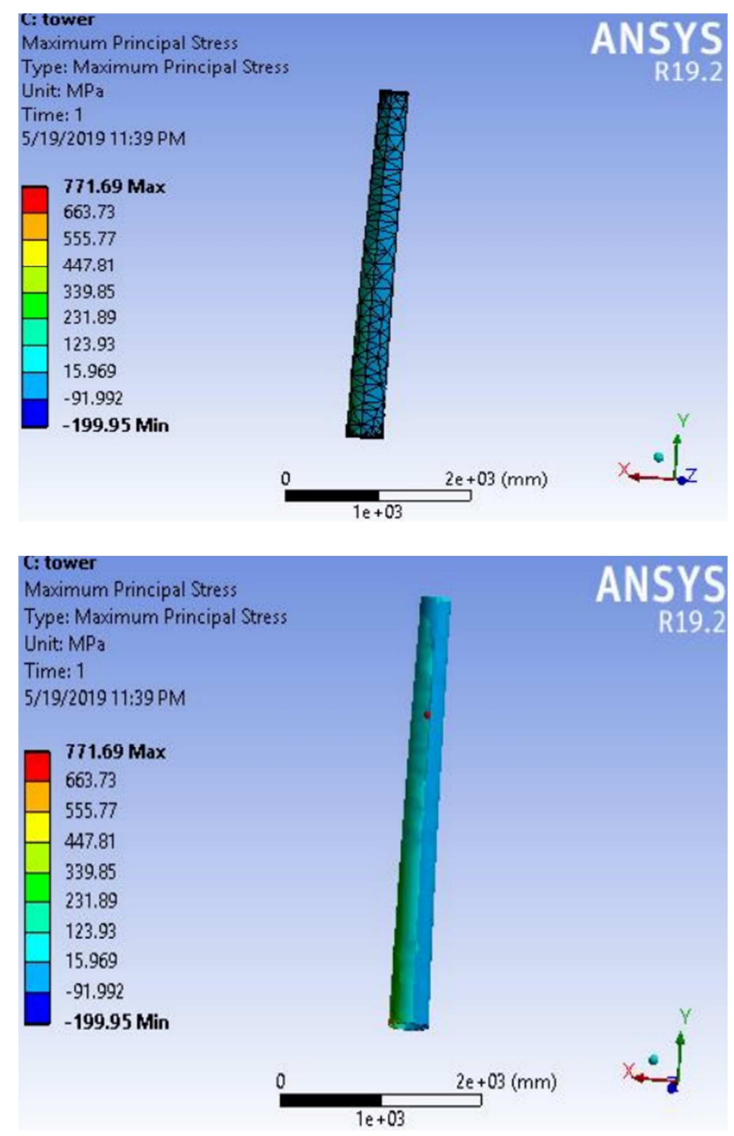

Figure 30. Tower maximum principal stress.

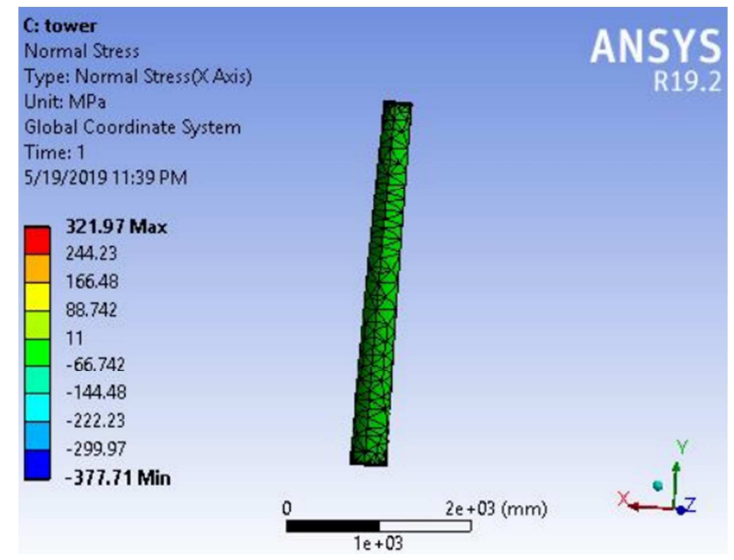

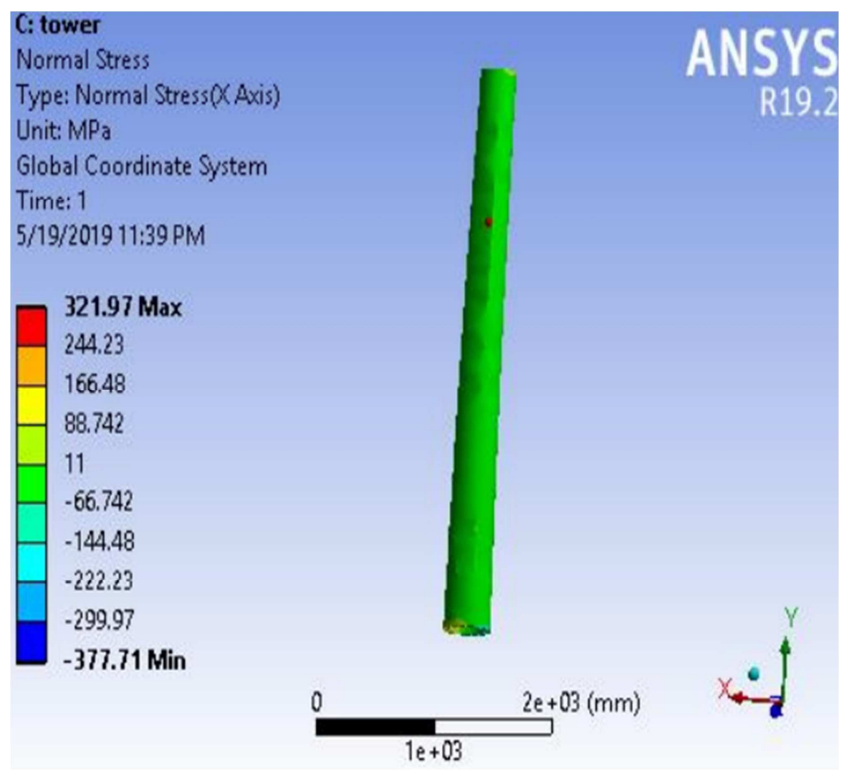

Figure 31. Tower bending stress.
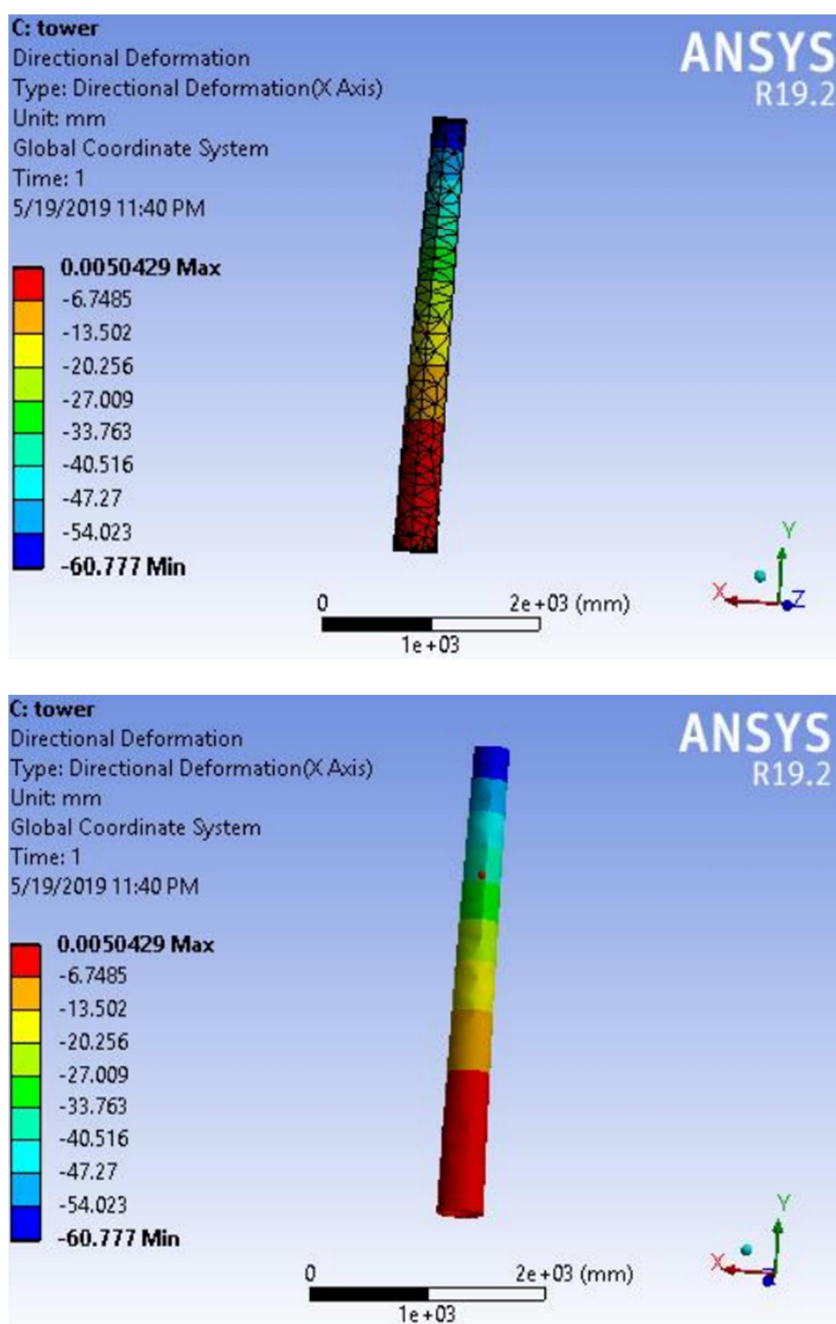

Figure 32. Tower directional deformation.

Fatigue analysis 


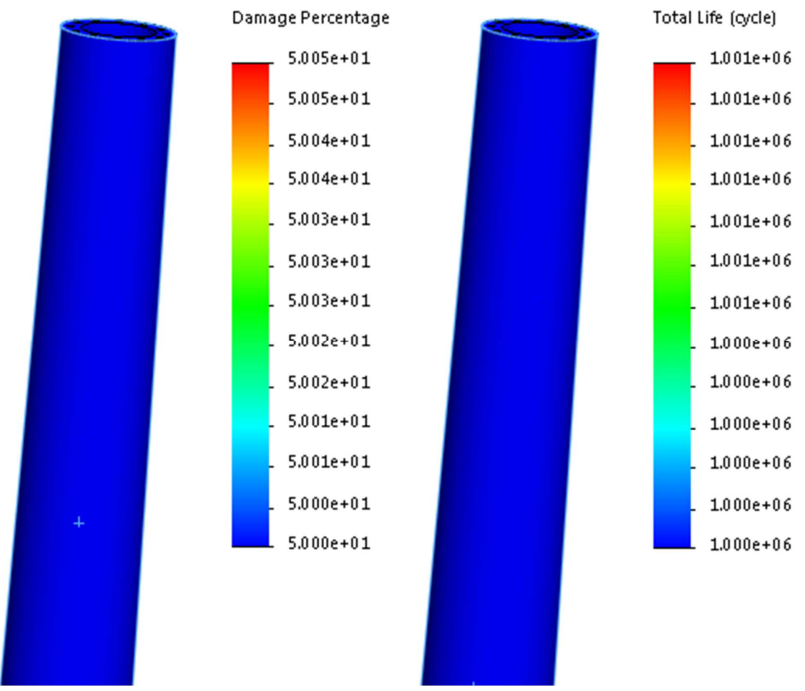

Figure 33. Damage percentage and total life.

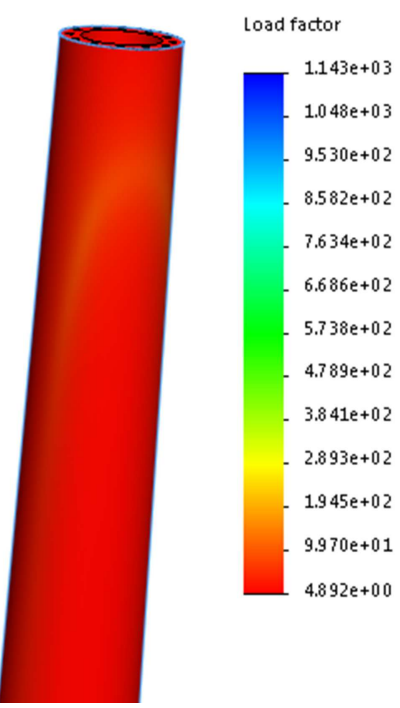

Figure 34. Load factor.

\subsection{Extreme Moment Analysis}

Table 7. Extreme moments.

\begin{tabular}{llll}
\hline No & Component & Governing equation & Result (KNm) \\
\hline 1 & Blade root extreme e torsion & $y=13.412 x^{2}+75.91 x$ & 205.5 \\
2 & Main shaft extreme torque & $y=132.6 x^{2}+2045.6 x$ & 4621.6 \\
3 & Resultant tower bottom moment & $y=378.06 x^{2}+2580.8 x$ & 6673.8 \\
4 & Tower top extreme torsion & $y=491.78 x^{2}+1884.9 x$ & 6589.8 \\
5 & Resultant blade flange moment & $y=282.91 x^{2}+2729.1 x$ & 6589.84 \\
\hline
\end{tabular}

Where $\mathrm{y}=$ extreme moment $(\mathrm{KNm}), \mathrm{x}=$ net rated power $(\mathrm{MW})$.

\subsection{Extreme Thrust Force}

Consider the time-varying or fatigue loads, which mechanically work the structure, particularly at the different attachment and component transitions throughout the turbine. Goodman diagrams are used to estimate fatigue life for various stress ratios experienced throughout the critical locations. These fluctuating loads often dictate the final sizing of structural members (beyond what is required for extreme loads). The utility scale WT sees more fatigue load cycles than any other manmade structure or machine many millions of cycles over its 20-year design life more than automobiles, ships, aircraft or rockets.

$$
y=18.371 x^{2}+217.07 x=507.624 K N
$$

Table 8. Mass calculation.

\begin{tabular}{|c|c|c|c|}
\hline No & Component & Governing Equation & Result (Tone) \\
\hline 1 & Tower & $y=7.7524 x^{2}+47.526 x$ & 126.06 \\
\hline 2 & Tower head with considering convertor & $y=4.8654 x^{2}+51.003 x$ & 119.952 \\
\hline 3 & Tower head w/t considering convertor & $y=4.8323 x^{2}+48.7961 x$ & 116.9214 \\
\hline 4 & Tower / meter & $y=0.0260 x^{2}+0.6976$ & 1.4992 \\
\hline 5 & Partial nacelle & $y=1.978 x^{2}+11.4378 x$ & 19.357 \\
\hline 6 & Gearbox & $y=1.6141 x^{2}+6.3934 x$ & 19.2432 \\
\hline 7 & All blades (with extender) & $y=0.4263 x^{2}+11.3307 x$ & 24.366 \\
\hline 8 & Partial hub & $y=0.3300 x^{2}+7.2729 x$ & 15.8658 \\
\hline 9 & Rotor mass & $y=18.453 x^{1.1357}$ & 40.5 \\
\hline 10 & Shaft & $y=0.2415 x^{2}+3.0699 x$ & 7.1058 \\
\hline 11 & Pitch & $y=0.1343 x^{2}+2.6526 x$ & 5.8424 \\
\hline 12 & Yaw & $y=0.1122 x^{2}+1.8548 x$ & 4.1584 \\
\hline 13 & Convertor & $y=-0.033 x^{2}+2.207 x$ & 4.2 \\
\hline 14 & Bearing & $y=0.1246 x^{2}+1.2623 x$ & 3.027 \\
\hline 15 & Generator & $y=-0.1304 x^{2}+3.5217 x$ & 6.5218 \\
\hline 16 & Each blade & $\mathrm{y}=-0.0317 x^{2}+3.8449 x-2.5952$ & $4.9678=5$ \\
\hline 17 & LV cable & $y=0.0519 x^{2}+1.2654 x$ & 2.7384 \\
\hline 18 & Bed plate (conventional) & $y=1.0242 x^{2}+4.5379 x$ & 13.1726 \\
\hline 19 & Machine head & $y=37.2765 x^{0.9815}$ & 73.5 \\
\hline 20 & Foundation & $y=35.1637 x^{2}+254.6463 x$ & 649.9474 \\
\hline 21 & Foundation + wind turbine & $y=50.6685 x^{2}+349.6983 x$ & 902.076 \\
\hline
\end{tabular}




\section{Conclusion}

Large horizontal axis wind turbine tower adopts approximate cylindrical shell structure, which determines the form of whole or partial buckling, is the main shape of damage to tower. The motive of buckling analysis of tower is to decide the buckling vital load and its corresponding modal and enhance anti-buckling capability. Tower buckling isn't always simplest associated with its load, however is likewise associated with its own form of shell structure. Cylindrical shell is high touchy disorder structure, and there are various styles of disorder for wind turbine tower, including opening, and thickness alternate of tower, which makes practical important buckling stress beneath the proper circumstance.

This paper presents the analytical, computer aided design of a horizontal axis wind turbine tower for a $2 \mathrm{MW}$ wind turbine for the Ethiopian wind site assessment information of pace and specific place altitude difference attention. Based on the analytical, static and dynamic analysis the designed wind turbine tower suggests a relevant widespread in terms of manufacturability and mechanical properties. The design tower is much less weight, cost powerful and static and dynamic solid tower.

Based on this successful structural design of the tower it is observed that it is efficient, safe and financial design of the complete wind turbine system. Also it provides easy access for maintenance of the rotor components and sub-components, and easy transportation and erection. Good designs ought to incorporate aesthetic features of the overall machine shape.

The design tower support the entire nacelle assembly along with the rotor above the ground level without any suffer of loading.

The buckling of $2 \mathrm{MW}$ horizontal axis wind turbine tower is studied by way of theoretical evaluation and numerical simulation. The buckling modal of tower under axial force, wind stress, bending moment and lateral pressure is simulated by means of ANSYS $\AA$ and MATLAB $\AA$ software program in above figures as shown. The effects show impact to buckling and other loading consequences on tower. Tower buckling is not only related to its load, but is also related to its own form of shell structure. Cylindrical shell is high sensitive defect structure, and there are various forms of defect for wind turbine tower, such as opening, and thickness change of tower, which makes practical critical buckling stress below the ideal condition.

\section{Acknowledgements}

I would first like to thank my supervisor, Belete Sirahbizu Yigezu (PhD) Assistant Professor and University-Industry Linkage Director; his workplace turned into continually open on every occasion I bumped into a trouble spot or had a question approximately my paper. He always allowed this paper to be my personal work and additionally for his valuable steerage and encouragement at some stage in this examine and thanks for his patience and trust throughout the study.

\section{References}

[1] David A. Spera, Wind Turbine Technology Fundamental Concepts in Wind Turbine Engineering, Second Edition 2009, ASME Press (American Society of Mechanical Engineers).

[2] James F. Manwell, Jon G. McGowan, Anthony L. Rogers Wind energy explained theory, design and application 2002, Wiley.

[3] Hansen, A. C. Butterfield, C. P., Aerodynamics of Horizontal Axis Wind Turbines, Annual Review of Fluid Mechanics, 1993, Vol. 25

[4] Le Gourieres, D., Wind Power Plants, Theory and Design, 1982, Pergamon Press.

[5] Durand, W. F., Aerodynamic Theory, Volume 2, 1963 Dover Publications.

[6] Glauert, H., The Elements of Airfoil and Airscrew Theory, 1983 Cambridge Univ. Press.

[7] Timmer, W. A., van Rooy, R. P. J. O. M., "Thick Airfoils for HAWTs", Journal of Wind Engineering and Industrial Aerodynamics, Vol. 39, 1992.

[8] Barlas, T.; Lackner, M. The Application of Smart Structures for Large Wind Turbine Rotor Blades. In Proceedings of the Iea Topical Expert Meeting; Delft University of Technology: Delft, The Netherlands, 2006.

[9] Ahlstrom, A. Emergency stop simulation using a finite element model developed for large blade deflections. Wind Energy 2006.

[10] Chandrala M., Choubey A. Gupta B., 2012. Aerodynamic analysis of horizontal axis wind turbine blade. Journal of Engineering Research and Application 2.

[11] Benini E., Toffolo A., 2005. Optimal design of horizontal-axis wind turbines using blade-element theory and evolutionary computation. Journal of Solar Energy Engineering.

[12] Adama I Wind farm Project Summary Report 2011.

[13] Case_study-ASHEGODA-WIND-FARM-rev-1.

[14] Ethiopia Wind Resource Poster Landscape WBESMAP Apr 20162.

[15] Ethiopian Electric Power Corporation (EEPCo). Strategic management and programming. Facts in brief; 2011.

[16] Wei Tong, wind power generation and wind turbine design, 2010 WIT press.

\section{Biography}

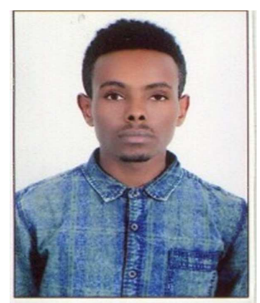

Gizachew Dereje Tsega (MSc) Lecturer, University of Gondar. 


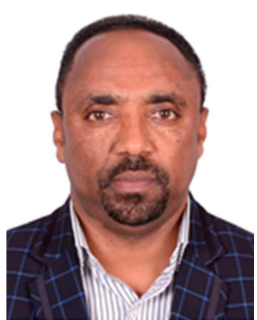

Belete Sirahbizu Yigezu (PhD) Assistant Professor and University-Industry Linkage Director Addis Ababa Science and Technology University. 